\title{
A Model of Delegated Project Choice*
}

\author{
Mark Armstrong \\ Department of Economics \\ University College London \\ London WC1E 6BT, UK
}

\author{
John Vickers \\ Department of Economics \\ University of Oxford \\ Oxford OX1 3UQ, UK
}

June 2008

\begin{abstract}
We present a model in which a principal delegates the choice of project to an agent with different preferences. The principal determines the set of projects from which the agent may choose. The principal can verify the characteristics of the project chosen by the agent, but does not know which other projects are available to the agent. Two frameworks are considered: (i) a static setting in which the collection of available projects is exogenous to the agent but uncertain, and (ii) a dynamic setting in which the agent searches for projects.
\end{abstract}

Keywords: Delegation, principal-agent, rules, search, merger policy.

\section{Introduction}

In this paper we present an analysis of a principal-agent problem in which the principal can influence the agent's behaviour not by outcome-contingent rewards but by specifying what the agent is, and is not, allowed to do. The agent, whose preferences differ from those of the principal, will select from her available projects the permitted project that best serves her interests. The principal can verify whether or not the selected project is indeed within the permitted set, but cannot observe the number or characteristics of the projects available to the agent. How then should the principal specify the set of projects from which the agent is permitted to choose?

Our analysis was originally motivated by a question in competition policy that is explained more fully in section 2: should mergers be permitted provided that they are

*This paper supersedes our 2007 paper entitled "A Model of Delegated Project Choice with Application to Merger Policy". We are grateful to V. Bhaskar, James Dow, Bengt Holmstrom, Michael Katz, Niko Matouschek, Meg Meyer, Barry Nalebuff, Eric Rasmusen, David Sappington, Karl Schlag, Aggey Semenov, Jidong Zhou, and to seminar participants at Barcelona, Bristol, Cambridge, Columbia, MIT, Oslo, Oxford, Singapore, Toulouse, UCL, Warwick and Yale for comments and discussion. Armstrong gratefully acknowledges the support of the Economic and Social Research Council (UK). 
expected to enhance (or at least not to diminish) "total welfare", or should the policy standard be in terms of consumer welfare? Even if the ultimate policy objective is total welfare, the answer is not obvious because the merger proposals brought forward by (profit-seeking) firms are selected by them from a wider pool of potential mergers. As we shall see, because of this selection effect, it could be that total welfare is higher on average under a consumer standard than a total welfare standard.

Our goal, however, is not to compare alternative ad hoc rules, such as the consumer and total welfare standards in the merger policy example. Rather, it is to characterize optimal permission sets in terms of the fundamentals of our models. Sometimes optimal permission rules are found to have strikingly simple forms. Two variants of our framework are analyzed. First is the model of project choice examined in section 3. This has a static setting in which the agent chooses one project from an exogenous, but uncertain, number of available projects. The principal optimally restricts agent choice in a way that forbids some projects that are good, in the uncertain hope of inducing the agent instead to choose a project that is better for the principal. This bias could be interpreted as strategically putting less weight on the agent's payoff than is in the true welfare function. The second is the model of project search analyzed in section 4 . This is in a dynamic setting where the agent can influence the uncertain arrival rate of projects by exerting costly effort. Here, the principal again may disallow some good projects but, in order to induce search effort, may allow some that are detrimental for his interests; in such examples the principal strategically puts more weight on the agent's payoff than is in his true welfare function. In both models we show how optimal permission sets vary with preferences, probability distributions, project numbers, discount rates, and so on.

The merger policy illustration that first motivated our work is but one example of constrained delegation in the absence of contingent payments between principal and agent. For instance, the framework could apply to decision making within a firm. A CEO of a company may, within limits, delegate investment project choice to a more junior manager, where the $\mathrm{CEO}$ is interested in shareholder value (for example), while the manager enjoys private benefits from certain projects and private costs from searching for projects. Our analysis suggests that, in such a setting, it will not generally be optimal for the CEO to approve all projects whose expected returns exceed the cost of capital. A quite different, behavioural, application of our model is to a single agent whose future tastes may differ from her current tastes. This agent may wish to constrain her future choices now, if feasible, in order to maximize her well-being over time.

More generally, our analysis addresses an aspect of the theory of optimal rules namely, the relationship between the ultimate objective of the rule-setter and the optimal rule to commit to. That relationship is not straightforward inasmuch as the likely consequences of a rule - including for the attainment of the ultimate objective depend on the responses of agents seeking to maximize, within the rules, their own objectives. The interplay between rules and the responses that they induce is at the heart of our analysis.

Some other papers have examined situations in which a principal delegates decisionmaking to a (potentially) better-informed agent whose preferences differ from those 
of the principal, and where contingent transfers between principal and agent are ruled out. ${ }^{1}$ Aghion and Tirole (1997) show how, depending on information structure and payoff alignment, it may be optimal for a principal to delegate full decision-making power to a potentially better-informed agent. The principal's loss of control over project choice can be outweighed by advantages in terms of encouraging the agent to develop and gather information about projects. In like vein Baker, Gibbons, and Murphy (1999), though they deny formal delegation of authority, examine informal delegation through repeated-game relational contracts. Even an informed principal able to observe project payoffs may refrain from vetoing ones that yield him poor payoffs in order to promote search incentives for the agent.

Our work is closer to the models which analyze constrained delegation, where the agent can make decisions but only within specified limits and the principal's problem is to decide how much leeway to give the agent. ${ }^{2}$ This literature was initiated by Holmstrom (1984), and the elements of his model go as follows. There is a set of projects, indexed by a scalar variable $d$ which takes values in some large interval $D$, of which one needs to be chosen. A given project generates payoffs to the two parties which depend on the state of the world, represented by $\theta$, and only the agent observes this parameter. The preferences of the principal and agent may differ, and if project $d$ is chosen when the state is $\theta$ the principal obtains payoff $Y_{P}(d, \theta)$ and the agent has payoff $Y_{A}(d, \theta)$. The principal's problem is to choose a permission set, say $\mathcal{D} \subset D$, in order to maximize his expected payoff (given his prior on the distribution of $\theta$ ), while predicting that the agent will choose her most preferred project in $\mathcal{D}$ given the state $\theta$. This "delegation problem" coincides with the "mechanism design problem" where the agent announces the state of the world, $\hat{\theta}$, and the principal commits to a (deterministic) decision rule $d(\hat{\theta})$ which maps the announcement to the implemented project. The two approaches are equivalent since the principal never directly observes the true $\theta$ and by making a suitable announcement $\hat{\theta}$ the agent can implement any project in the range of the rule $d(\cdot)$.

Holmstrom mostly limits attention to the (often realistic) situation in which the permission set is an interval. Subject to this assumption (and other regularity conditions), he shows that an agent whose preferences are closer to the principal's will be given more discretion. (This result has subsequently sometimes been termed the "ally principle".) Following Holmstrom's initial contribution, subsequent papers have analyzed when interval delegation is in fact the optimal delegation policy, making the additional assumption that $\theta$ is a scalar variable. ${ }^{3}$ Melumad and Shibano (1991) were

\footnotetext{
${ }^{1}$ One plausible reason why monetary incentives are not always given to an agent is that the agent in practice performs several tasks, and giving incentives to do one task well might induce the agent to under-perform on other, unmeasured, aspects of her job. See Holmstrom and Milgrom (1991) for further discussion of this point, as well as section 1 of Alonso and Matouschek (2008) for further discussion about why it is worthwhile to analyze situations without monetary incentives.

${ }^{2}$ In an experimental study, Falk and Kosfeld (2006) find that agents can be de-motivated when their choices are restricted by the principal, a factor we do not take account of in our model.

${ }^{3}$ Szalay (2005) presents an interesting variant on this delegation problem in which interval delegation is often sub-optimal. In his model, there is no divergence in preferences between the principal and agent, but the agent incurs a private cost to observe $\theta$. He shows that it can be optimal for the principal to remove intermediate projects from $\mathcal{D}$ so that the agent is forced to choose between
} 
the first to calculate optimal permission sets, in the special case where preferences were quadratic, where the agent had a known bias, and where $\theta$ was uniformly distributed. They found that interval delegation was optimal when principal and agent have ideal projects which are similarly responsive to the state $\theta$, but that otherwise it could be optimal to have "holes" in $\mathcal{D}$. Martimort and Semenov (2006) find a sufficient condition on the distribution of $\theta$ for interval delegation to be optimal. Alonso and Matouschek (2008) systematically investigate when interval delegation is optimal, and they generalize Melumad and Shibano's insight that the relative responsiveness of preferred decisions to the state is the key factor for this. One interesting finding is that when interval delegation is sub-optimal the ally principle need not hold and an agent with preferences more aligned with those of the principal might optimally be given less discretion. ${ }^{4}$

Those models in the Holmstrom tradition differ from ours in respect of project specification and the form of asymmetric information. In particular, they characterize each project by a scalar parameter, all projects are always feasible, and the agent has private information about a payoff-relevant state of the world. In our model, by contrast, payoffs of the chosen project to both principal and agent are known, but only a finite collection of projects is feasible and only the agent knows what those projects are. Like the papers discussed above, our aim is to characterize the optimal delegation set for the principal to allow the agent to choose from, but in the twodimensional setting where the principal can observe both his own and the agent's payoff from the project chosen by the agent. ${ }^{5}$

\section{Welfare Standards in Merger Policy}

An important debate in antitrust policy concerns the appropriate welfare standard to use when deciding whether to prohibit a merger (or some other form of conduct). ${ }^{6}$ The two leading contenders are a total welfare standard, where mergers are evaluated

relatively extreme options, and this sharpens the agent's incentive to discover $\theta$. The benefit from this can outweigh the principal's loss due to the possibility that the ideal project given the realized state is forbidden.

${ }^{4}$ Kovác and Mylovanov (2007) discuss the impact of stochastic delegation schemes, in which the agent chooses from a constrained set of lotteries over projects rather than from a subset of projects themselves. They show, broadly speaking, that when interval delegation is optimal in Alonso and Matouschek's deterministic framework no extra benefit is generated by stochastic schemes, but otherwise the principal can sometimes do better by offering lotteries over projects.

${ }^{5} \mathrm{~A}$ recent paper which also investigates a two-dimensional delegation problem is Amador, Werning, and Angeletos (2006). There, an agent with quasi-hyperbolic preferences has wealth which she consumes over two periods. If there were no uncertainty about her preferences, she would gain by committing to a fixed consumption path at time zero. However, she will receive a utility shock in period 1 and this uncertainty gives a motive allow some flexibility in consumption. Amador et al. show that the time-zero agent limits her consumption possibilities to a subset of feasible consumption paths, and they find a condition which implies that the optimal delegation set simply involves placing a ceiling on first-period consumption.

${ }^{6}$ In practice, many mergers giving rise to competition concerns are permitted subject to conditions (e.g., asset disposals) rather than prohibited altogether. Such merger remedies are beyond the scope of the illustrative example discussed here. 
according to whether they decrease the unweighted sum of producer and consumer surplus, and a consumer welfare standard, where mergers detrimental to consumers are blocked. Many economic commentators feel that antitrust policy should aim to maximize total welfare, whereas in most jurisdictions the focus is more on consumer welfare alone. See Farrell and Katz (2006) for an excellent overview of the issues.

One purpose of this paper is to examine a particular strategic reason, discussed by Lyons (2002) and Fridolfsson (2007), to depart from the regulator's true welfare standard, which is that a firm may have a choice of merger possibilities. A less profitable merger might be better for total welfare, but will not be chosen under a total welfare standard. To illustrate, consider Figure 1, which is similar to those presented in section IV.B in Farrell and Katz (2006). ${ }^{7}$

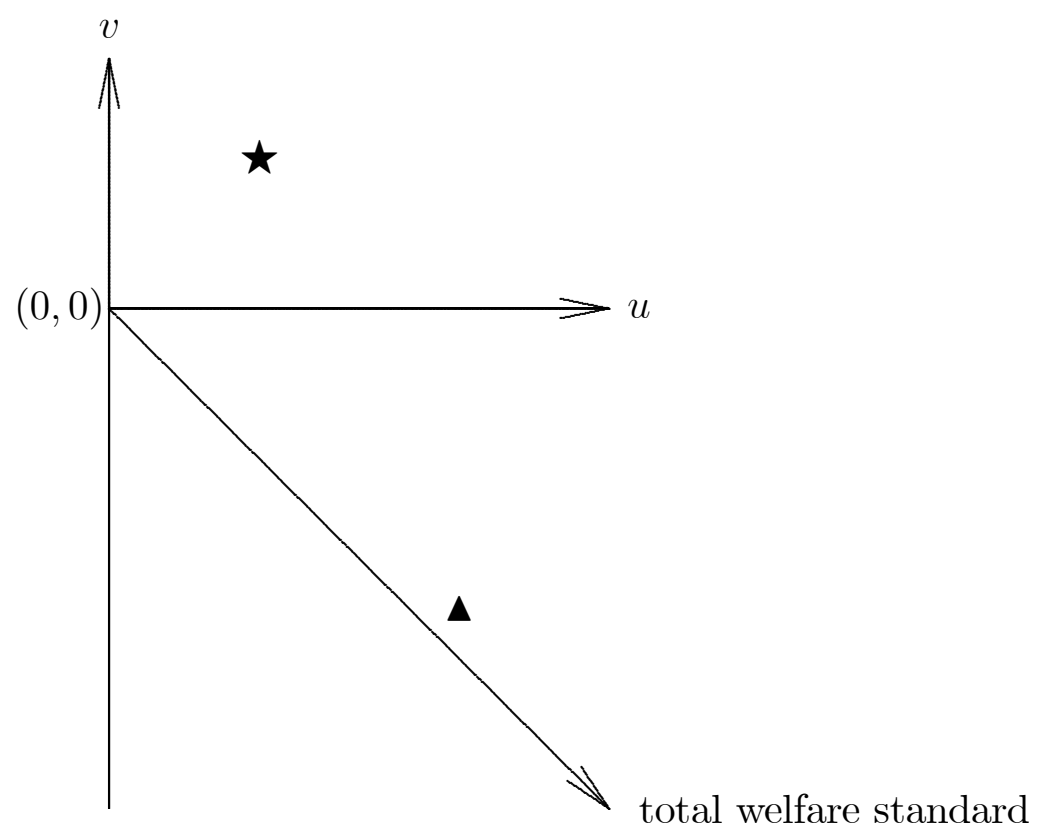

Figure 1: The Impact of Welfare Standard on Chosen Mergers

Here, $u$ represents the gain in total profit resulting from a merger, while $v$ measures the resulting net gain (which may be negative) to consumers. Suppose that $u$ and $v$ are verifiable once a merger is proposed to the competition authority. If the regulator follows a total welfare standard, he will permit any merger which lies above the negatively-sloped line in the figure. Suppose the firm has two mergers to choose from, depicted by $\boldsymbol{\Lambda}$ and $\star$ on the figure. With a total welfare standard, the firm will choose the merger with the higher $u$ payoff, i.e., the $\boldsymbol{\Delta}$ merger. However, the regulator would prefer the alternative $\star$ since that yields higher total welfare. If the regulator instead imposed a consumer welfare standard, so that only those mergers which lie above the horizontal line $v=0$ are permitted, then the firm will be forced to choose the preferred merger. In this case, a regulator wishing to maximize total

\footnotetext{
${ }^{7}$ The discussion in Farrell and Katz (2006) is a "reduced-form" version of the formal models in Lyons (2002) and Fridolfsson (2007).
} 
welfare is better off if he imposes a consumer welfare standard. As Farrell and Katz (2006, page 17) put it: "if we want to maximize gains in total surplus (northeasterly movements as shown in Figure 1) and firms always push eastwards, there is something to be said for someone adding a northerly force."

Nevertheless, there is a potential cost to adopting a consumer welfare standard: if the $\boldsymbol{\Delta}$ merger turns out to be the only possible merger then a consumer welfare standard will not permit this even though the merger will improve total welfare. Thus, the choice of welfare standard will depend on the number of possible mergers and the distribution of profit and consumer surplus gains for a possible merger. For instance, as Farrell and Katz observe, if efficiency gains from a merger take the form of reductions in fixed, not marginal, costs, any merger between competitors can only cause reductions in consumer surplus and so a consumer welfare standard would forbid all such mergers (including those which increase total welfare).

\section{Choosing a Project}

A principal ("he") delegates the choice of project to an agent ("she"). Both parties are risk-neutral. There may be several projects for the agent to choose from, although only one can be implemented over the relevant time horizon. We will consider two variants of the delegated choice problem: (i) a static setting in which the agent can choose one project from an exogenous but random number of available projects (as analyzed in this section); and (ii) a dynamic search model in section 4 in which the agent can choose the arrival rate of potential projects.

A project is fully described by two parameters, $u$ and $v$. The agent's payoff if the type- $(u, v)$ project is implemented is $u$, while the principal's payoff is $v+\alpha u$. Here, $\alpha \geq 0$ represents the weight the principal places on the agent's interests, and $v$ represents factors specific to the principal's interests. ${ }^{8}$ In the merger context, $\alpha=1$ when the antitrust authority wishes to maximize total welfare and $\alpha=0$ when it cares only about consumers. More generally, the case $\alpha=1$ applies in delegation settings where the principal hires the agent for a fixed fee, not contingent on results, before the agent knows which projects are available. ${ }^{9}$

Each project is an independent draw from the same distribution for $(u, v)$. Since the agent will never propose a project with a negative payoff, without loss of generality we suppose that only non-negative $u$ are realized. The marginal density of $u \geq 0$ is $f(u)$. The conditional density of $v$ given $u$ is denoted $g(v, u)$ and the associated conditional distribution function is $G(v, u)$. Here, $v$ can be positive or negative. For technical reasons, suppose that the support of $(u, v)$ is a rectangle $\left[0, u_{\max }\right] \times$ $\left[v_{\min }, v_{\max }\right]$. In particular, the support of $v$ given $u$ does not depend on $u$. Suppose also that $v_{\min } \leq 0 \leq v_{\max }$, so that $(0,0)$ lies in the support of $(u, v)$. Finally, suppose that both $f$ and $g$ are twice continuously differentiable and non-zero on the support

\footnotetext{
${ }^{8}$ It would also be straightforward to allow the agent to care about the principal's welfare.

${ }^{9}$ Specifically, the principal chooses the permission set $\mathcal{D}$ and transfer $t$ to maximize $E[v+\alpha u]-$ $(1-\alpha) t$ subject to $E[u]+t \geq u_{0}$, the agent's outside option. Optimality requires that $\mathcal{D}$ maximizes $E[v]+E[u]$.
} 
of $(u, v)$.

The principal delegates the choice of project to the agent. We assume that it is impossible, or not credible, for the principal to give monetary incentives to the agent to choose a desirable project. Once the agent selects a particular project from the set of possible projects, that project's characteristics are verifiable. ${ }^{10}$ The class of mechanisms we analyze is as follows: before the agent has any private information, the principal commits to a (measurable) "permission set" (or delegation set) of projects, denoted $\mathcal{D} \subset\left[0, u_{\max }\right] \times\left[v_{\min }, v_{\max }\right]$, and the agent can then implement any project she chooses in $\mathcal{D}$. (See section 3.3 for discussion of our approach and the impact of more ornate delegation schemes.)

In this first model, the number of projects is random and the probability that the agent has exactly $n \geq 0$ possible projects is $q_{n}$. (Our analysis applies to the case where there are surely $N$ projects, so that $q_{N}=1$, but the analysis is no easier for that case.) The realization of $(u, v)$ for each project is described by $f$ and $g$ as above, and is independent for each project among the $n$ projects. In addition, $(u, v)$ is distributed independently of $n$.

For each $u$, let

$$
\mathcal{D}_{u}=\{v \text { such that }(u, v) \in \mathcal{D}\}
$$

be the set of $u$-projects which are permitted, and let

$$
p(u)=\int_{v \in \mathcal{D}_{u}} g(v, u) d v
$$

to be the proportion of type- $u$ projects which are permitted. Let

$$
x(u)=1-\int_{u}^{u_{\max }} p(z) f(z) d z
$$

to be the probability that any given project either has agent payoff $z$ less than $u$ or is not permitted. Note that

$$
x^{\prime}(u)=p(u) f(u) .
$$

(Our assumptions ensure that $x(\cdot)$ is differentiable.) If there are exactly $n \geq 1$ available projects, the probability that the agent's preferred permitted project has payoff no higher than $u$ is $(x(u))^{n}$, and so the density of the agent's preferred permitted project is

$$
\frac{d}{d u}(x(u))^{n}=n p(u) f(u)(x(u))^{n-1}
$$

(One of the $n$ projects must be permitted and give agent payoff $u$, which has probability $p(u) f(u)$, while the remaining $n-1$ projects must either have agent payoff lower than $u$ or not be permitted.) Summing over $n$ implies that the density of the highest- $u$ permitted project is

$$
\frac{d}{d u} \sum_{n=0}^{\infty} q_{n}(x(u))^{n} .
$$

\footnotetext{
${ }^{10}$ What is needed here is that the principal and agent have symmetric information about the project characteristics when the project is chosen. The actual outcome of the project could still be uncertain at this point, in which case $u$ and $v$ represent the expected payoffs to the two parties.
} 
If we write $\phi(x) \equiv \sum_{n=0}^{\infty} q_{n} x^{n}$ for the probability generating function (or PGF) associated with the random variable $n$, it follows that the density of the agent's preferred permitted project is $\frac{d}{d u} \phi(x(u))$. Useful properties of PGFs which we will sometimes use is that they are well-defined on the interval $0 \leq x \leq 1$ and convex and increasing over this interval.

The principal's payoff with the permission set $\mathcal{D}$ is

$$
\begin{aligned}
\int_{0}^{u_{\max }}\left\{E\left[v \mid u \text { and } v \in \mathcal{D}_{u}\right]+\alpha u\right\} \frac{d}{d u} \phi(x(u)) d u \\
=\int_{0}^{u_{\max }}\left\{\int_{v \in \mathcal{D}_{u}} v g(v, u) d v+\alpha u p(u)\right\} \phi^{\prime}(x(u)) f(u) d u .
\end{aligned}
$$

The principal's problem is to maximize expression (2) taking into account the relationship between and $p$ and $x$ in (1) and the endpoint constraint $x\left(u_{\max }\right)=1$. The following lemma shows that the optimal permission set takes the following "threshold" form:

Lemma 1 In the optimal policy there exists a threshold rule $r(\cdot)$ such that

$$
(u, v) \in \mathcal{D} \text { if and only if } v \geq r(u) \text {. }
$$

Proof. From (1), the function $x(\cdot)$ depends on $\mathcal{D}$ only via the "sufficient statistic" $p(u)$, not on the particular $v$-projects which are permitted given $u$. Therefore, for any candidate function $p(u)$ the principal might as well permit those particular $v$-projects which maximize the term $\{\cdot\}$ in the $(2)$, subject to the constraint that the proportion of type- $u$ projects is $p(u)$. But the problem of choosing the set $\mathcal{D}_{u}$ in order to

$$
\operatorname{maximize} \int_{v \in \mathcal{D}_{u}} v g(v, u) d v \text { subject to } \int_{v \in \mathcal{D}_{u}} g(v, u) d v=p(u)
$$

is solved by permitting the projects with the highest $v$ so that the proportion of permitted projects is $p(u)$, i.e., that $\mathcal{D}_{u}=\{v$ such that $v \geq r(u)\}$ for some $r(u)$.

Thus, the problem simplifies to the choice of threshold function $r(\cdot)$ rather than the choice of permission set $\mathcal{D}$. (A similar argument is valid in the search model presented in section 4.) Figure 2 depicts a possible threshold function $r(\cdot)$, and also shows $x(u)$ depicted as the measure of the shaded area.

It is useful to introduce one further piece of notation, and define

$$
V(r, u) \equiv E[v \mid u \text { and } v \geq r]
$$

to be the expected value of $v$ given that the project has agent payoff $u$ and that $v$ is at least $r$. Recasting (2) in terms of $r(\cdot)$ rather than $\mathcal{D}$, the principal's problem is to choose $r(\cdot)$ to maximize

$$
\int_{0}^{\infty}[V(r(u), u)+\alpha u][1-G(r(u), u)] f(u) \phi^{\prime}(x(u)) d u
$$




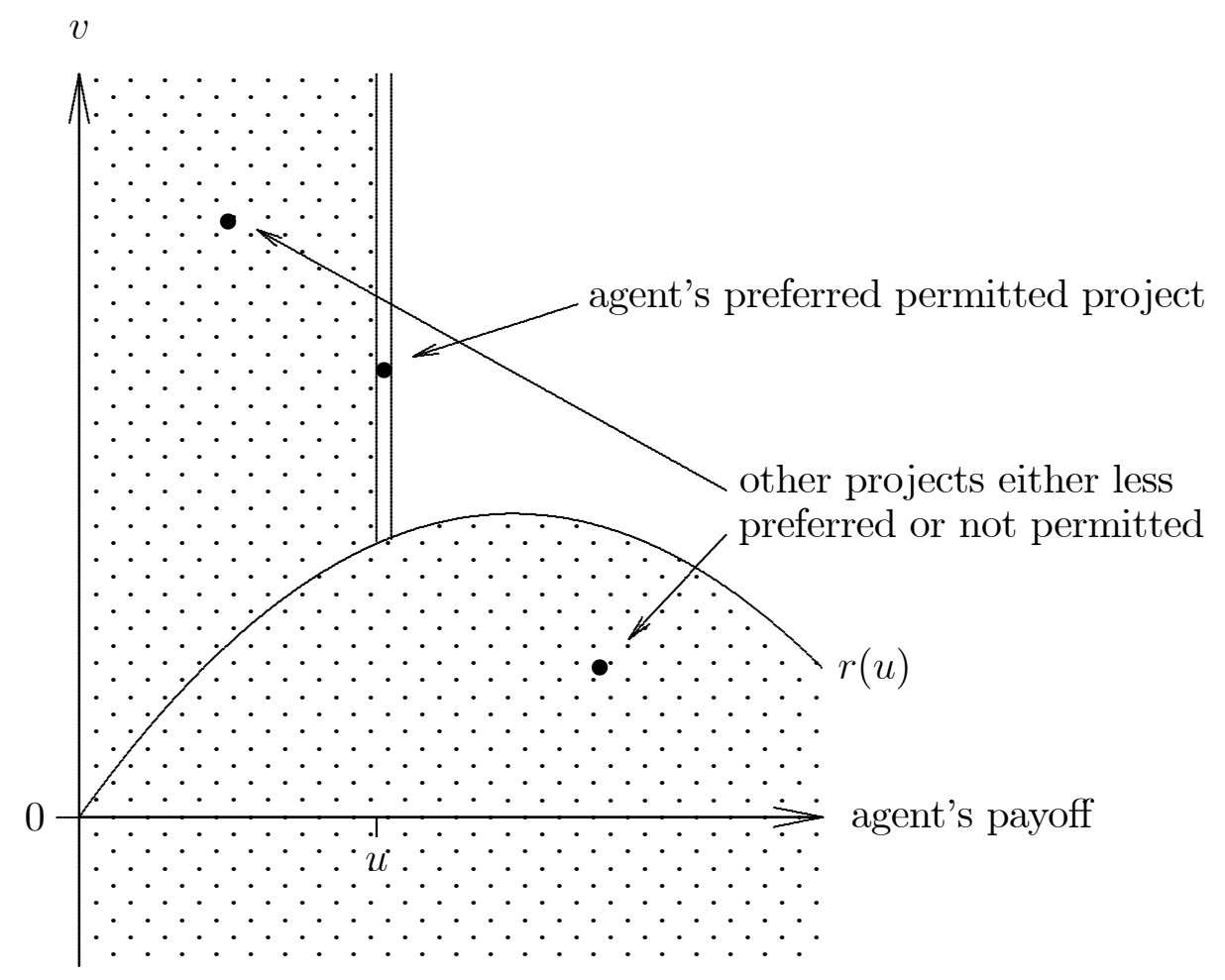

Figure 2: The Agent's Preferred Permitted Project

subject to the "equation of motion"

$$
x^{\prime}(u)=f(u)[1-G(r(u), u)]
$$

and the endpoint condition $x\left(u_{\max }\right)=1$.

This classical calculus of variations problem is solved formally in the appendix, but its solution can be understood intuitively with the following argument. Consider some point $(u, r(u))$ on the frontier of the permitted set. For the set to be optimal it must be that the principal is indifferent between his payoff $[r(u)+\alpha u]$ at that point and his expected payoff from the agent's next-best permitted alternative, conditional on the agent's best permitted project being $(u, r(u))$.

To calculate the latter expected payoff, note that the density that a project drawn at random has payoffs $(u, r(u))$ is $f(u) g(r(u), u)$. Then the probability that one out of $n$ projects has payoffs $(u, r(u))$ and all the others have agent utility no greater than $z \leq u$ or are not permitted is $n f(u) g(r(u), u)[x(z)]^{n-1}$. Taking the $q_{n}$-weighted sum across $n$, the probability that one project has payoffs $(u, r(u))$ and all other permitted projects have utility no greater than $z$ is therefore $f(u) g(r(u), u) \phi^{\prime}(x(z))$. In particular, the probability that the agent's preferred permitted project is $(u, r(u))$ is $f(u) g(r(u), u) \phi^{\prime}(x(u))$. Conditional on that event, the probability that the nextbest permitted alternative for the agent has agent utility no greater than $z \leq u$ is

$$
\frac{f(u) g(r(u), u) \phi^{\prime}(x(z))}{f(u) g(r(u), u) \phi^{\prime}(x(u))}=\frac{\phi^{\prime}(x(z))}{\phi^{\prime}(x(u))},
$$


which has associated density $\phi^{\prime \prime}(x(z)) x^{\prime}(z) / \phi^{\prime}(x(u))$. Therefore the indifference condition is

$$
r(u)+\alpha u=\frac{1}{\phi^{\prime}(x(u))} \int_{0}^{u}[V(r(z), z)+\alpha z] \phi^{\prime \prime}(x(z)) x^{\prime}(z) d z
$$

for all $u \in\left[0, u_{\max }\right]$.

In particular, we see from (5) that $r(0)=0$. This implies that the principal does not wish to restrict the desirable projects available to the agent whose best project has only zero payoff, i.e., there is "no distortion at the bottom". The reason for this is that when $u=0$ there is no strategic benefit to restricting choice. (The strategic effect of raising $r(u)$ above $-\alpha u$ is to increase the probability that the agent will choose a smaller $z$, and this effect cannot operate when $u=0$.) Differentiating (5) with respect to $u$ and using (4) implies the Euler equation for the principal's problem, which is expression (6) below.

Proposition 1 The optimal threshold rule satisfies the Euler equation

$$
r^{\prime}(u)+\alpha=[V(r(u), u)-r(u)][1-G(r(u), u)] f(u) \frac{\phi^{\prime \prime}(x(u))}{\phi^{\prime}(x(u))}
$$

with initial condition $r(0)=0$, terminal condition $x\left(u_{\max }\right)=1$, and equation of motion (4). A sufficient condition for the second-order condition for the calculus of variations problem to be satisfied is that

$$
\zeta(x) \equiv \frac{\phi^{\prime \prime}(x)}{\phi^{\prime}(x)} \text { weakly decreases with } x .
$$

Proof. See appendix.

Expression (6) reveals that $\zeta$ in (7) is important for the form of the solution. A short list of examples for this term includes:

- If $n$ is known to be exactly $N \geq 1$ for sure (so $q_{N}=1$ ), then $\phi(x)=x^{N}$ and $\zeta(x)=(N-1) / x$.

- If $n$ is Poisson with mean $\mu$ (so $q_{n}=e^{-\mu} \frac{\mu^{n}}{n !}$ for $\left.n \geq 0\right)$ then $\phi(x)=e^{-\mu(1-x)}$ and $\zeta(x) \equiv \mu$.

- If $n$ is Binomial (the sum of $N$ Bernoulli variables with success probability $a$ ) then $\phi(x)=(1-a(1-x))^{N}$ and $\zeta(x)=a(N-1) /(1-a(1-x))$. The "known $n$ " case is a special case of the Binomial with $a=1$. The Poisson is a limit case of the Binomial when $a N=\mu$ and $a \rightarrow 0$.

- If $n$ is Geometric (so $q_{n}=(1-a) a^{n-1}$ for $n \geq 1$ and some parameter $a \in(0,1)$ ) then $\phi(x)=(1-a) x /(1-a x)$ and $\zeta(x)=2 a /(1-a x)$.

- If $n=2$ with probability $\pi$ and $n=1$ with probability $1-\pi$, then $\phi(x)=$ $(1-\pi) x+\pi x^{2}$ and $\zeta(x)=1 /(k+x)$ where $k=(1-\pi) /(2 \pi)$. 
Assumption (7) is valid for the Binomial distribution - and hence for the "known $n$ " and Poisson sub-cases - and for the $n \in\{1,2\}$ example, but not for the Geometric distribution.

Define the "naive" threshold rule to be

$$
r_{\text {naive }}(u)=-\alpha u \text {. }
$$

This is the threshold rule which permits all desirable projects, i.e., those projects such that $v+\alpha u \geq 0$. This rule might be implemented by a principal who ignored the strategic effect that the agent will only choose the project with the highest $u$ whenever she has a choice. As such, the naive rule is optimal for the principal when the agent never has a choice of project, i.e., when $q_{0}+q_{1}=1$. (In this case $\phi^{\prime \prime} \equiv 0$, the right-hand side of $(6)$ vanishes, and so $r(\cdot) \equiv r_{\text {naive }}(\cdot)$ is optimal.)

Outside this dull case, though, the right-hand side of (6) is strictly positive. Therefore, since $r^{\prime}(u)+\alpha>0$ and $r(0)=0$ it follows that $r(u)>r_{\text {naive }}(u)$ when $u>0$. Therefore, the principal forbids some strictly desirable projects (and never permits undesirable projects). Moreover, the gap between the optimal and the naive rule, $r(u)-r_{\text {naive }}(u)$, strictly increases. We state this formally as:

Corollary 1 Suppose the agent sometimes has a choice of project (i.e., $q_{0}+q_{1}<1$ ). Then it is optimal for the principal to forbid some strictly desirable projects, and the gap between the optimal threshold rule $r(u)$ and the naive threshold rule $r_{\text {naive }}(u)$ widens with $u$. In particular, when $\alpha=0$ the optimal threshold rule increases with $u$.

What is the intuition for why the principal wishes to exclude some desirable projects from the permitted set, whenever the agent sometimes has a choice of project? Suppose the principal initially allows all desirable projects, so that $r(u) \equiv$ $r_{\text {naive }}(u)$. If the principal increases $r(\cdot)$ slightly at some $u>0$, the direct cost is approximately zero, since the principal then excludes projects about which he is almost indifferent (since $r(u)+\alpha u=0$ ). But there is a strictly beneficial strategic effect: there is some chance that the agent's highest- $u$ project is excluded by the modified permitted set, in which case there is a chance that she chooses another project which is permitted, say with $z<u$. This alternative project is unlikely to be marginal for the principal, and instead the principal will expect to get payoff $V(r(z), z)+\alpha z$, which is strictly positive when $r(z)=-\alpha z$. This argument indicates that it is beneficial to restrict desirable projects, and not to permit undesirable projects. Moreover, it is intuitive that the strategic effect is more important for higher $u$, since it applies over a wider range $z<u$, and this explains why the gap $r(u)-r_{\text {naive }}(u)$ widens with $u$.

Corollary 1 shows that the gap between the optimal threshold rule and the naive threshold rule widens with $u$. What else can we say about the shape of $r(\cdot)$ ? Write

$$
I(r, u)=[V(r, u)-r][1-G(r, u)]=\int_{r}^{v_{\max }}[v-r] g(v, u) d v=\int_{r}^{v_{\max }}[1-G(v, u)] d v .
$$

Then $I_{r}=-[1-G(r, u)]<0$. Differentiating expression (6) with respect to $u$ shows that

$$
\frac{r^{\prime \prime}}{r^{\prime}+\alpha}=\frac{I_{r}}{I} r^{\prime}+\frac{I_{u}}{I}+\frac{f^{\prime}}{f}+\frac{\zeta^{\prime}}{\zeta} x^{\prime}=\frac{I_{r}}{I}[I f \zeta-\alpha]+\frac{I_{u}}{I}+\frac{f^{\prime}}{f}-\frac{\zeta^{\prime}}{\zeta} f I_{r} .
$$


Here, the second equality follows from (4) and (6). Thus, since $r^{\prime}+\alpha$ is positive we have

$$
r^{\prime \prime} \stackrel{\text { sign }}{=} \underbrace{f I_{r}\left[\zeta-\frac{\zeta^{\prime}}{\zeta}\right]}_{[i]}+\underbrace{\left[-\alpha \frac{I_{r}}{I}\right]}_{[i i]}+\underbrace{\frac{I_{u}}{I}}_{[i i i]}+\underbrace{\frac{f^{\prime}}{f}}_{[i v]} .
$$

Note that $\left[\zeta-\zeta^{\prime} / \zeta\right]$ in term $[i]$ in expression (9) is positive if and only if $\phi^{\prime \prime} /\left(\phi^{\prime}\right)^{2}$ decreases with $x$. This is a weak condition, satisfied by all five of our examples of PGFs above. (It is weaker than assumption (7) above.) If this holds, then term $[i]$ is negative. Term $[i i]$ is positive, unless $\alpha=0$ when it is zero. Term [iii] is zero if $u$ and $v$ are independently distributed, and it is positive if the two parameters are positively correlated (in the sense that $G(v, u)$ decreases in $u$ ). Term $[i v]$ has the sign of $f^{\prime}$, and in particular this is zero when $u$ is uniformly distributed.

Thus we see that whether $r$ is (locally) convex or concave depends on the sum of four terms. In some special cases we can $\operatorname{sign} r^{\prime \prime}$. For instance, if $u$ has weakly decreasing density and is independent from $v$, and if $\alpha$ is close to zero, then $r(\cdot)$ is concave (and increasing). More generally, (9) shows that $\alpha$ large and the existence of positive correlation between $u$ and $v$ are both forces which tend to make $r$ convex (in which case the permission set $\mathcal{D}$ is a convex set).

We next discuss comparative statics for this problem. First, we show that a greater fraction of projects should be permitted when more weight is placed on the agent's interests.

Proposition 2 Let $\alpha_{L}$ and $\alpha_{H}$ be two weights placed by the principal on the agent's payoff, where $\alpha_{L}<\alpha_{H}$. Let $r_{i}(\cdot)$ and $x_{i}(\cdot)$ solve the Euler equation (6) when $\alpha=\alpha_{i}$ for $i=L, H$. If assumption (7) holds then $x_{L}(0)>x_{H}(0)$, i.e., the fraction of permitted projects increases with $\alpha$.

Proof. Condition (6) implies that at $u=0$ and any other $u$ such that $r_{L}(u)=r_{H}(u)$

$$
\frac{r_{L}^{\prime}(u)+\alpha_{L}}{r_{H}^{\prime}(u)+\alpha_{H}}=\frac{\zeta\left(x_{L}(u)\right)}{\zeta\left(x_{H}(u)\right)} .
$$

If $x_{L}(0) \leq x_{H}(0)$, then by assumption $(7) \zeta\left(x_{L}(0)\right) \geq \zeta\left(x_{H}(0)\right)$, and so (10) implies that $r_{L}^{\prime}(0)>r_{H}^{\prime}(0)$. In particular, $r_{L}(u)>r_{H}(u)$ for small $u>0$. If $x_{L}(0) \leq x_{H}(0)$ then $r_{L}(\cdot)$ must cross $r_{H}(\cdot)$ at some point. (If $r_{L}$ were uniformly above $r_{H}$ then clearly the fraction of prohibited projects with $\alpha_{L}$ would be greater than with $\alpha_{H}$.) Let $u^{*}$ be the first point above zero where the curves cross. In particular, we must have $r_{L}^{\prime}\left(u^{*}\right) \leq r_{H}^{\prime}\left(u^{*}\right)$. In addition, we must have $x_{H}\left(u^{*}\right) \geq x_{L}\left(u^{*}\right)$ since $x_{H}(0) \geq x_{L}(0)$ and $r_{H}(u) \leq r_{L}(u)$ for $u \leq u^{*}$. But then (10) implies that

$$
1>\frac{r_{L}^{\prime}\left(u^{*}\right)+\alpha_{L}}{r_{H}^{\prime}\left(u^{*}\right)+\alpha_{H}}=\frac{\zeta\left(x_{L}\left(u^{*}\right)\right)}{\zeta\left(x_{H}\left(u^{*}\right)\right)} \geq 1
$$

a contradiction. We deduce that the curves can never cross, and so our initial assumption $x_{L}(0) \leq x_{H}(0)$ cannot hold. 
Thus we see that the more the principal cares about the utility of the agent, the more discretion - in the sense of a greater fraction of projects being permitted - the agent is given. This result is similar to the "ally principle" in the Holmstrom-type model mentioned in section 1, where the more likely the agent's preferences were to be close to the principal's, the more discretion the agent was given. It is also simple to show that the agent's expected payoff with the principal's optimal scheme increases in $\alpha .^{11}$

It is also natural to expect that when the agent is likely to have more projects to choose from, the principal will further constrain the permitted set of projects. With more projects available, the agent is likely to have at least one which lies close to the principal's preferred project. There is a close connection between "having more projects" and the function $\zeta(x)$ being shifted upwards. For instance, in our five examples of distributions for $n$ above, this is true. (In the Poisson case, $\zeta$ is just equal to the expected number of projects.) More generally, a natural interpretation of "having more projects" is the following: suppose the number of agent's projects is initially governed by the PGF $\phi_{L}(\cdot)$, and suppose the agent then has access to an additional source of projects which has PGF $\phi(\cdot)$, say. A fundamental property of PGFs is that the PGF corresponding to the independent sum of two random variables is the product of the underlying PGFs. Therefore, the PGF for the agent with these two sources of projects is just $\phi_{H}=\phi \phi_{L}$. Under a regularity condition, $\phi_{H}^{\prime \prime} / \phi_{H}^{\prime}$ is greater than $\phi_{L}^{\prime \prime} / \phi_{L}^{\prime}$ :

Lemma 2 Suppose that $1 / \phi_{L}(x)$ is a strictly convex function, and let $\phi_{H}(x)=$ $\phi(x) \phi_{L}(x)$ for any PGF $\phi(\cdot)$ except the trivial PGF $\phi \equiv 1$. Let $\zeta_{i}=\phi_{i}^{\prime \prime} / \phi_{i}^{\prime}$ for $i=L, H$. Then

$$
\zeta_{H}(x)>\zeta_{L}(x)
$$

Proof. Condition (11) is equivalent to the condition that $\phi_{H}^{\prime}(x) / \phi_{L}^{\prime}(x)$ strictly increases with $x$. But

$$
\frac{\phi_{H}^{\prime}}{\phi_{L}^{\prime}}=\frac{\phi^{\prime} \phi_{L}+\phi \phi_{L}^{\prime}}{\phi_{L}^{\prime}}=\phi+\phi^{\prime} \frac{\phi_{L}}{\phi_{L}^{\prime}}
$$

and so

$$
\frac{d}{d x} \frac{\phi_{H}^{\prime}}{\phi_{L}^{\prime}}=\phi^{\prime \prime} \frac{\phi_{L}}{\phi_{L}^{\prime}}+\phi^{\prime}\left[2-\frac{\phi_{L} \phi_{L}^{\prime \prime}}{\left(\phi_{L}^{\prime}\right)^{2}}\right] \geq \phi^{\prime}\left[2-\frac{\phi_{L} \phi_{L}^{\prime \prime}}{\left(\phi_{L}^{\prime}\right)^{2}}\right] .
$$

Therefore, provided that $\phi^{\prime}>0$ (which is the case whenever there is sometimes an additional project), a sufficient condition for (11) to hold is that $2\left(\phi_{L}^{\prime}\right)^{2}>\phi_{L} \phi_{L}^{\prime \prime}$, which is equivalent to the requirement that $1 / \phi_{L}$ be strictly convex.

Note that the condition that $1 / \phi_{L}(x)$ is convex is satisfied in all our listed examples of PGFs above. Using this notion of "more projects", we can obtain the following result. ${ }^{12}$

\footnotetext{
${ }^{11}$ When $\alpha=\alpha_{H}$ the principal prefers $r_{H}(u)$ to $r_{L}(u)$ and vice versa when $\alpha=\alpha_{L}$. It follows by the principal's revealed preference that expected $u$ is higher with $\alpha_{H}$ than $\alpha_{L}$.

${ }^{12}$ An example where adding more projects widens the optimal set of permitted projects is the following. Suppose initially the agent has no projects at all with probability $1-\varepsilon$ and exactly two
} 
Proposition 3 Suppose the number of projects is initially $n_{L}$ with $P G F \phi_{L}(x)$ which satisfies (7) and such that $1 / \phi_{L}$ is convex. Suppose next that the agent has access to a second source of projects with number $n$ (where $n$ is positive with some positive probability), and now the total number of projects is the independent sum $n_{H}=n_{L}+n$. Let $r_{i}(\cdot)$ and $x_{i}(\cdot)$ solve the Euler equation (6) when the number of projects is $n_{i}$ for $i=L, H$. Then $x_{H}(0)>x_{L}(0)$, i.e., the fraction of permitted projects decreases when there are more projects.

Proof. From Lemma 2, the PGF $\phi_{H}$ associated with $n_{H}$ satisfies (11). The rest of the proof follows the argument (with $L$ and $H$ permuted) of Proposition 2.

Note that we use the term "having more projects" to mean that the agent now has the independent sum of her original projects and a supply of other projects. This is a stronger requirement than the more usual notion of first-order stochastic dominance. Indeed, it is possible to find examples (even in the most regular cases) where stochastic dominance leads to a smaller fraction of projects being excluded.

Without making further assumptions, it is hard to make more progress in characterizing the solution to (6). This expression is in general a second-order differential equation in $x, x^{\prime}$ and $x^{\prime \prime}$ (see expression (43) in the appendix). However, there are two cases in which the Euler equation can simplify to a first-order equation. First, when $v$ is independently distributed from $u$ the Euler equation simplifies and with further assumptions (for instance when $\alpha=0$ ), the Euler equation becomes a firstorder equation in $x$ and $x^{\prime}$. Second, when $n$ is a Poisson variable, the dependence on $x(u)$ in the Euler equation vanishes, and (6) becomes a first-order equation in $r$ and $r^{\prime}$. These special cases are discussed in more detail in the next two sub-sections.

\subsection{Independent distributions}

Suppose in this section that the distribution of $v$ is independent of $u$. In particular, in the following we write $g(v), G(v), V(r)$ and $I(r)$ as functions which do not depend on $u$. Then the Euler equation can then be simplified in the following manner. ${ }^{13}$

Lemma 3 If the distribution for $v$ is independent of $u$, expression (6) is equivalent to

$$
\frac{d}{d u}\left[\frac{V(r(u))-r(u)}{f(u)} \frac{d}{d u} \phi(x(u))\right]=\frac{\alpha}{f(u)} \frac{d}{d u} \phi(x(u)) .
$$

projects with probability $\varepsilon$. Because the state when no projects materialize plays no role in the determination of $r(\cdot)$, the optimal threshold rule for this agent is just as if there were two projects for sure. Such a threshold rule will strictly exclude some desirable projects. Consider next the situation in which the agent has exactly one more project than the previous situation (i.e., $n=1$ with probability $1-\varepsilon$ and $n=3$ with probability $\varepsilon$ ). Whenever $\varepsilon$ is small, the state where there is only one project will dominate the choice of $r(\cdot)$, and almost all desirable projects will be permitted, thus widening the set of permitted projects. One can check that the PGF for the original distribution for $n$ does not satisfy the requirement that $1 / \phi_{L}$ is convex.

${ }^{13}$ Within the calculus of variations literature, this transformation of the Euler equation is known as the Beltrami identity. 
Proof. Note that

$$
-I^{\prime}(r)=1-G(r)=\frac{x^{\prime}(u)}{f(u)},
$$

and

$$
V(r)-r=\frac{I(r)}{-I^{\prime}(r)} .
$$

Multiplying both sides of (6) by $-I^{\prime}(r(u)) \phi^{\prime}(x(u))$ and using (13) it follows that (6) is equivalent to

$$
-I^{\prime}(r(u)) \phi^{\prime}(x(u))\left[r^{\prime}(u)+\alpha\right]=I(r(u)) \phi^{\prime \prime}(x(u)) x^{\prime}(u) .
$$

Therefore, we have

$$
\begin{aligned}
-\alpha I^{\prime}(r(u)) \phi^{\prime}(x(u)) & =I(r(u)) \phi^{\prime \prime}(x(u)) x^{\prime}(u)+I^{\prime}(r(u)) \phi^{\prime}(x(u)) r^{\prime}(u) \\
& =\frac{d}{d u}\left[I(r(u)) \phi^{\prime}(x(u))\right] \\
& =\frac{d}{d u}\left[[V(r(u))-r(u)] \frac{x^{\prime}(u)}{f(u)} \phi^{\prime}(x(u))\right] \\
& =\frac{d}{d u}\left[\frac{V(r(u))-r(u)}{f(u)} \frac{d}{d u} \phi(x(u))\right]
\end{aligned}
$$

And

$$
-\alpha I^{\prime}(r(u)) \phi^{\prime}(x(u))=\alpha \frac{x^{\prime}(u)}{f(u)} \phi^{\prime}(x(u))=\frac{\alpha}{f(u)} \frac{d}{d u} \phi(x(u)) .
$$

Thus, (6) implies expression (12) and vice versa.

Inspecting (12) shows there are at least three cases in which this second-order differential equation can be integrated to a first-order equation:

- when $\alpha=0$ the equation becomes

$$
[V(r(u))-r(u)] \frac{d}{d u} \phi(x(u))=k f(u)
$$

for some positive constant $k$;

- when $u$ is uniformly distributed the equation becomes

$$
[V(r(u))-r(u)] \frac{d}{d u} \phi(x(u))=k_{1} \phi(x(u))+k_{2}
$$

for two constants $k_{1}$ and $k_{2}$, and

- when $v$ is exponentially distributed, $V(r)-r$ is constant and so the equation becomes

$$
\frac{d}{d u} \log \left[\frac{1}{f(u)} \frac{d}{d u} \phi(x(u))\right]=\text { constant . }
$$


In the following discussion we examine further the first of these cases, where $\alpha=0$. Here, the principal obtains the same payoff with all density functions $f(\cdot)$ for $u$. To see this, change variables in (14) from $u$ to $F(u)$. That is to say, write $\hat{r}(F(u)) \equiv r(u)$ and $\hat{x}(F(u)) \equiv x(u)$, so that $\hat{r}$ represents the threshold rule expressed in terms of the cumulative fraction of $u$-projects $F$. Then (14) becomes

$$
[V(\hat{r}(F))-\hat{r}(F)] \frac{d}{d F} \phi(\hat{x}(F)) \equiv k,
$$

with initial condition $\hat{r}(0)$ and terminal condition $\hat{x}(1)=1$. In particular, the optimal threshold rule $\hat{r}(\cdot)$ does not depend on the distribution for $u$ at all, as long as $u$ is continuously distributed. ${ }^{14}$ Since the random variable $F(u)$ is uniformly distributed, it follows from expression (9) and a mild regularity condition on $\zeta(x)$ that $\hat{r}(\cdot)$ is an increasing and concave function of $F(u)$. In sum, with $\alpha=0$ and $u$ and $v$ independent the principal's payoff does not depend on the distribution of $u$. As such, only ordinal rankings of $u$ matter for the principal.

We note that when $\alpha=0$, although the principal does not care directly about the agent's payoff, he nevertheless hopes that the agent has a permitted project with high $u$. In particular, the principal's expected payoff conditional on $u$, namely $V(r(u))$, increases with $u$. It follows that, even without re-optimizing the threshold rule $r(\cdot)$, the principal's expected payoff increases if the agent has more projects (in the sense of having an additional source of projects) because projects from the additional source will be chosen only if they have higher $u$ than what would otherwise have been chosen.

\subsection{Poisson distribution for the number of projects}

If $n$ follows a Poisson distribution with mean $\mu$, the Euler equation (6) becomes a first-order differential equation in $r(u)$ :

$$
r^{\prime}(u)+\alpha=\mu[V(r(u), u)-r(u)][1-G(r(u), u)] f(u) .
$$

The comparative statics of $r(\cdot)$ with respect to $\alpha$ and $\mu$ are stronger than the corresponding results in the general setting reported above in Propositions 2 and 3 :

Proposition 4 With a Poisson distribution for the number of available projects, the optimal threshold rule $r(\cdot)$ is pointwise increasing in $\mu$ and decreasing in $\alpha$.

Proof. The method is similar the proof of Proposition 2. Consider first the impact of increasing $\mu$, and let $\mu_{L}$ and $\mu_{H}>\mu_{L}$ be two values for $\mu$. Let $r_{L}(\cdot)$ and $r_{H}(\cdot)$ be the corresponding optimal threshold rules. From (16) it follows that at $u=0$ and any other $u$ such that $r_{L}(u)=r_{H}(u)$

$$
\frac{r_{L}^{\prime}(u)+\alpha}{r_{H}^{\prime}(u)+\alpha}=\frac{\mu_{L}}{\mu_{H}}<1,
$$

\footnotetext{
${ }^{14}$ Note that this argument requires us to change variables in expression (14), and so $F(u)$ needs to be differentiable and, in particular, the distribution for $u$ has no "atoms". If there were atoms, then we would need to consider what project the agent would choose in the event of a "tie", when there would two projects which yielded the same maximal agent payoff $u$.
} 
so $r_{L}^{\prime}(u)<r_{H}^{\prime}(u)$ at all such $u$. So $r_{H}$ can never cross $r_{L}$ from above. We deduce that $r_{H}(u)>r_{L}(u)$ for all $u>0$. The argument for the impact of $\alpha$ on $r(\cdot)$ is similar.

Moreover, it is straightforward to show from (16) that

$$
\begin{aligned}
& \left.r(u) \rightarrow v_{\max } \text { as } \mu \rightarrow \infty \text { (if } u>0\right), \text { and } \\
& r(u) \rightarrow r_{\text {naive }}(u)=-\alpha u \text { as } \mu \rightarrow 0 .
\end{aligned}
$$

Thus, with the ability to choose from many projects, the agent is essentially given no discretion, and only those projects with the highest payoff to the principal are permitted. In addition, as it becomes unlikely that the agent has any choice of project, the principal should allow all desirable projects to be implemented.

While solution to equation (16) can easily be solved numerically - and we present some examples of this at the end of this section - it apparently cannot be solved analytically without making further assumptions. One natural simplification is to make the differential equation homogeneous, so that there is no direct dependence on $u$ in (16). This is done by supposing (i) that $u$ is uniformly distributed on, say, $[0,1]$ and (ii) that $u$ and $v$ are independent. In this case, (16) becomes

$$
r^{\prime}=\mu I(r)-\alpha
$$

where $I(\cdot)$ is given in (8).

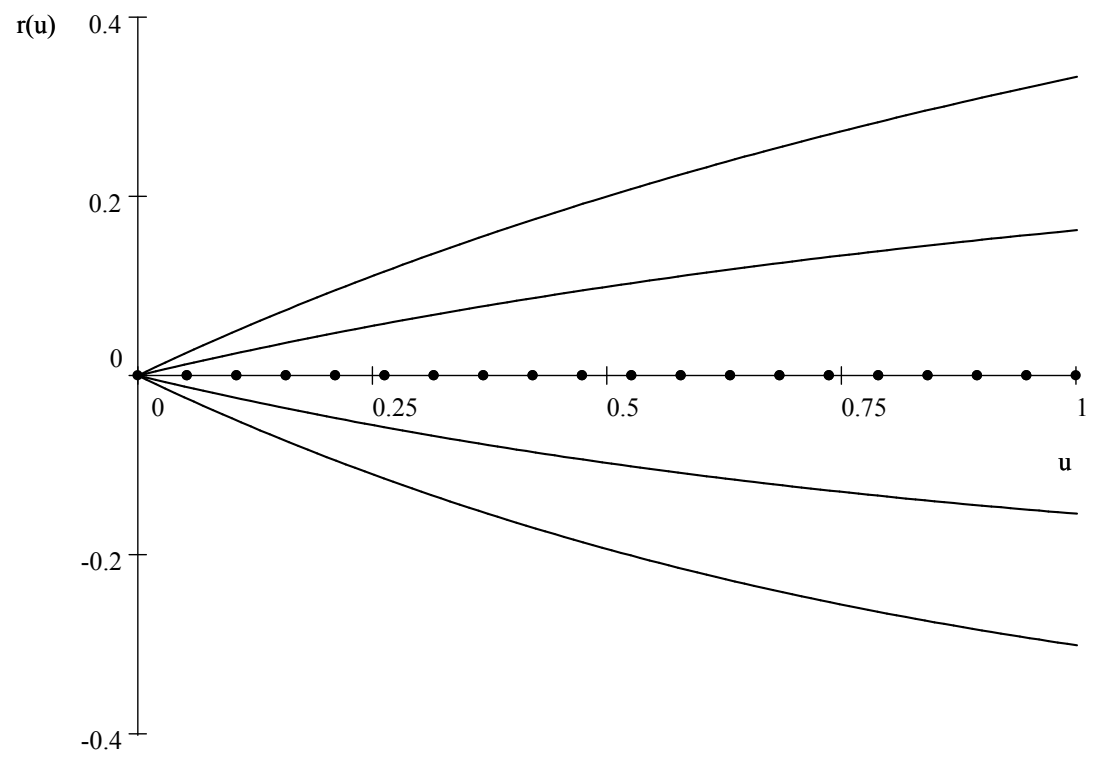

Figure 3: Uniform-Poisson example with $\mu=2$ and $\alpha=0, \frac{1}{4}, \frac{1}{2}$ (dotted), $\frac{3}{4}$ and 1

Note that if $\alpha=\mu I(0)$, then the solution to (17) is simply the flat rule $r(u) \equiv 0$. Thus, in the merger context, if the regulator wishes to maximize total welfare (so $\alpha=1)$, then if the expected number of mergers is such that $\mu I(0)=1$ the regulator should optimally enforce a pure consumer welfare standard. Outside this knife-edge case, the solution to (17) will be increasing (decreasing) in $u$ when $\alpha<(>) \mu I(0)$. 
Moreover, by differentiating (17), it follows that $r(u)$ is concave (convex) when $\alpha<$ $(>) \mu I(0)$. Specifically, the solution to (17) when $\alpha \neq \mu I(0)$ is given implicitly by

$$
\int_{0}^{r(u)} \frac{1}{\mu I(r)-\alpha} d r=u .
$$

To illustrate some solutions to (18), suppose that $(u, v)$ is uniformly distributed on $[0,1] \times[-1,1]$ so that $I(r)=\frac{1}{4}(1-r)^{2}$. When $\alpha=0$, expression (18) implies that

$$
r(u)=\frac{\mu u}{4+\mu u} .
$$

When $\alpha>0$ expression (18) can be integrated (using partial fractions) to give

$$
r(u)=\left(1-\frac{4 \alpha}{\mu}\right) \frac{e^{u \sqrt{\alpha \mu}}-1}{(1+\sqrt{4 \alpha / \mu}) e^{u \sqrt{\alpha \mu}}-(1-\sqrt{4 \alpha / \mu})} .
$$

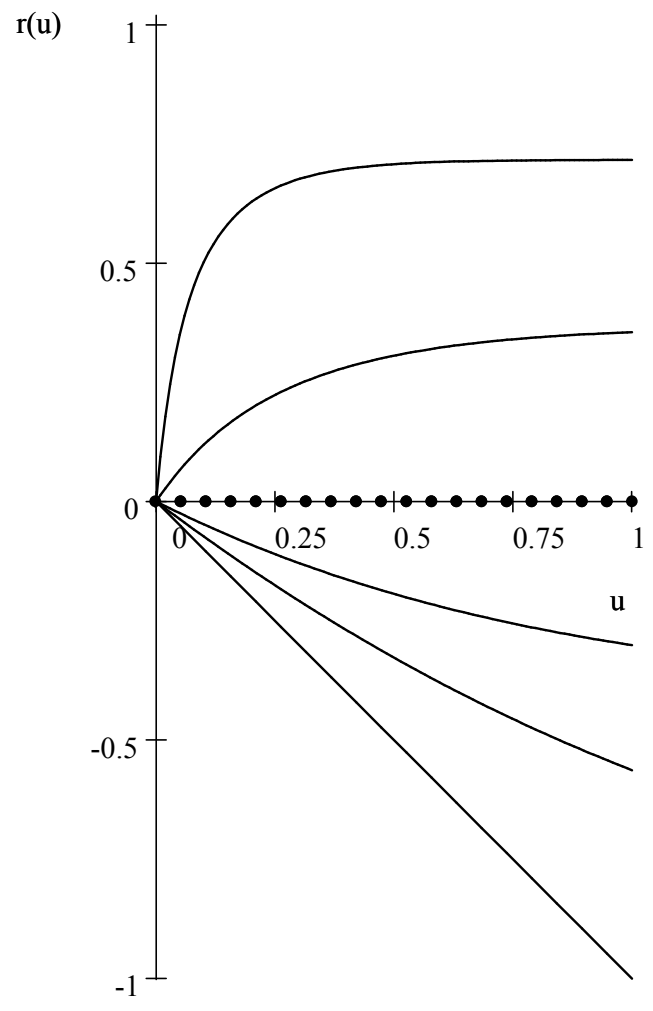

Figure 4: Uniform-Poisson case with $\alpha=1$ and $\mu=0,1,2,4$ (dotted), 10 and 50

As already mentioned, when $\mu=4 \alpha$ it is optimal in the Uniform-Poisson example to have the flat rule that only projects with positive $v$ are permitted. For $\mu>4 \alpha$, the solution is increasing and concave, while for $\mu<4 \alpha$ the solution is decreasing and convex. We depict these solutions in Figure 3 for fixed $\mu=2$ and various values for $\alpha$. Here, higher values of $\alpha$ correspond to lower threshold rules $r(u)$ (illustrating Proposition 4). Notice that a higher value of $\alpha$ is more likely to lead to $r(\cdot)$ being 
convex, as was indicated in general in expression (9). Figure 4 shows the solutions for fixed $\alpha=1$ and for various $\mu$. Here, higher curves correspond to higher $\mu$, which is consistent with Proposition 4. The straight line depicted for $\mu=0$ is just the naive rule which permits any desirable project.

The final issue we discuss here is the impact of correlation between $u$ and $v$ on the set of permitted projects. Intuitively, all else equal we expect that positive correlation between the two payoffs to lead to the agent being given more discretion: the agent can be trusted to pick projects with high $u$, and with correlation this tends to lead to high- $v$ projects too. To investigate this issue, suppose that $\alpha=0$ and $v$ is exponentially distributed with support $v \in[0, \infty)$ and mean $\lambda(u)$ given $u$, so that $G(v, u)=1-e^{-v / \lambda(u)}$. The variables $u$ and $v$ are independent if $\lambda$ is constant, they are positively correlated if $\lambda(u)$ is increasing in $u$, and they are negatively correlated if $\lambda(u)$ decreases.

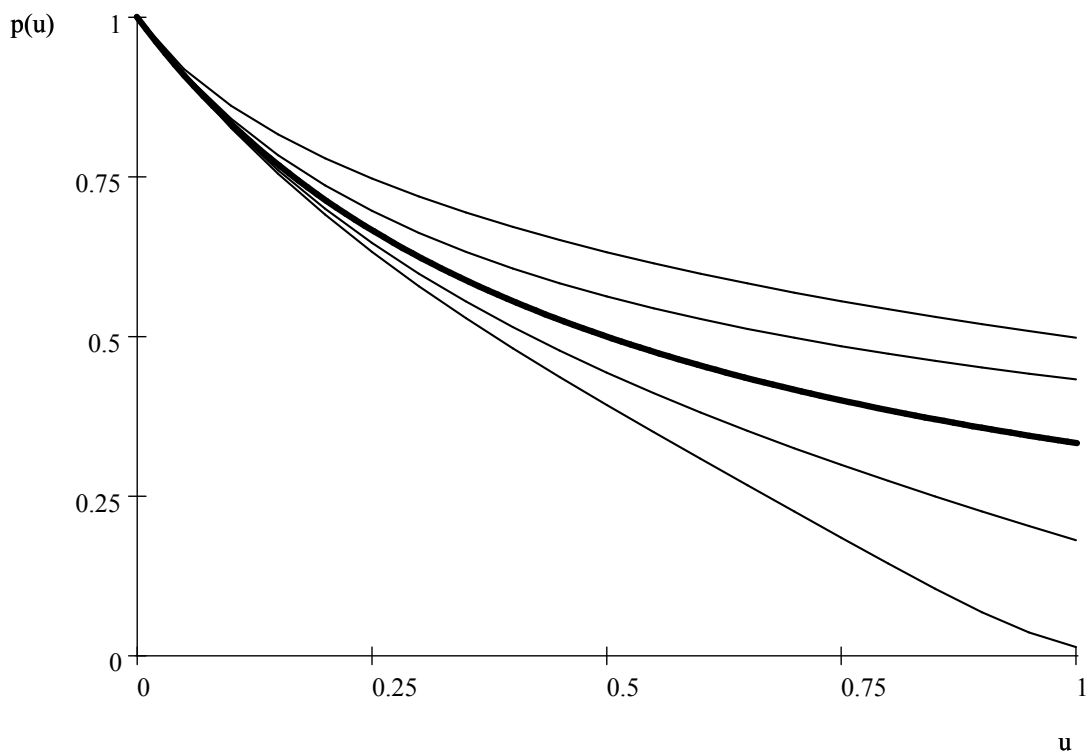

Figure 5: $p(u)$ when $\lambda(u)=1+k\left(u-\frac{1}{2}\right)$, for $k=-\frac{3}{2},-\frac{3}{4}, 0$ (in bold), $\frac{3}{4}$ and $\frac{3}{2}$

In this case $V(r, u)-r=\lambda(u)$, and so equation (16) becomes

$$
r^{\prime}(u)=\mu \lambda(u) f(u) e^{-\frac{r(u)}{\lambda(u)}} .
$$

If $\lambda$ is constant (the independence case), it follows from (21) that

$$
r(u)=\lambda \log (1+\mu F(u)) ; p(u)=\frac{1}{1+\mu F(u)} .
$$

If there is correlation, equation (21) does not seem explicitly soluble, but it is easily solved numerically.

Figure 5 depicts five solutions. In each of these we have set $\mu=2, u$ is uniform on $[0,1]$, and $\lambda(u)=1+k\left(u-\frac{1}{2}\right)$. (It follows that the unrestricted expectation of $v$ 
is the same equal to 1 - for all $k$, and so changes in the threshold rule for different $k$ are not simply due to changes in the mean of $v$.) A positive $k$ represents positive correlation between $u$ and $v$. Here, we illustrate the permission probabilities, $p(u)$, instead of the permission rules $r(u)$ illustrated on previous figures. In the figure the thick line depicts the independent case with $k=0$, and higher curves correspond to more positive (or less negative) correlation. Thus we see that more correlation is indeed associated with more discretion - in the sense that any type- $u$ project is more likely to be permitted - being granted to the agent. ${ }^{15}$

\subsection{Discussion of our delegation scheme}

In the analysis above we restricted attention to situations where the principal simply determines a permission set $\mathcal{D}$ for the agent to choose from. In this regard it is important first to emphasize the assumption here, as in delegation problems more generally, of commitment. The principal excluded some desirable projects from the permission set. But in the absence of commitment power, if the agent reports that she has no permitted project, the principal may have an incentive to "renegotiate", and to widen the set of permitted projects. In section 4 we find cases with the opposite bias, in which the principal permits some projects which are undesirable to him ex post, and this again requires commitment power to be credible. ${ }^{16}$

Second, we restricted attention to deterministic mechanisms, rather than, say, assigning to each proposed project a (possibly interior) probability $\pi(u, v)$ of being implemented. We did this in part because it is hard to imagine being able to commit to or implement a stochastic mechanism in practice. In any event, by the argument in Lemma 1, any stochastic scheme is dominated by the deterministic scheme giving rise to the same $p(u)$ function if the agent retains the alternative projects if her initial choice is (randomly) rejected. If, however, those alternatives are lost, it is possible that commitment to a stochastic scheme could do better for the principal than a deterministic scheme. ${ }^{17}$

Third, it may be asked whether, if feasible, more ornate deterministic delegation schemes (still without outcome-contingent payments) could improve the principal's expected payoff. Recall from the Introduction that in the Holmstrom (1984) setting the "delegation problem" coincided with the "mechanism design problem" in which the agent reports her (scalar) private information. In our setting her private information consists of the number and nature of available projects, and the mechanism design approach involves the agent announcing a list of projects and the principal

\footnotetext{
${ }^{15}$ The threshold rules $r(u)$ are not strictly nested in this example, and cross when $u$ is close to 1 .

${ }^{16}$ See Baker, Gibbons, and Murphy (1999) and Alonso and Matouschek (2007) for analyses of how commitment power can be endogenously generated with repeated interaction.

${ }^{17}$ For example, suppose that $\alpha=0$, that $n=2$ for sure, and that $(u, v)=(0.5,1)$ with probability 0.5 and $(u, v)=(0.9,0.1)$ with the same probability. In this case, the optimal deterministic scheme only permits the principal's favoured project, $(0.5,1)$. However, permitting the project $(0.9,0.1)$ with probability 0.5 yields the principal a higher expected payoff than banning it altogether: in both cases the agent would choose the principal's preferred project if she could, but if that is not available the stochastic scheme would still allow some chance of a desirable project being chosen.
} 
choosing to implement one project from the list. Two variants of this approach can be considered.

For instance, if the principal is able to verify the characteristics only of the chosen project (e.g., if auditing can only happen ex post), then the mechanism design problem again boils down to the delegation problem of simply choosing a set $\mathcal{D}$ of permitted projects. ${ }^{18}$ To see this, note that the set of projects can be partitioned into two subsets: those projects which, by making suitable reports of other projects, are chosen for implementation under the principal's decision rule, and those projects which are never implemented by the principal's rule. Faced with this rule, the agent will simply choose its preferred available project in the former set (if any), and announce any other projects required to implement that choice. Clearly, this mechanism is equivalent to the delegation problem where the agent can directly choose a project in this set. So if the principal can verify the characteristics only of the chosen project, our approach is optimal among the class of deterministic schemes.

If, however, the principal has wider powers of observation, more ornate schemes might be able to do better. For instance, consider the scenario in which the principal can cheaply verify the characteristics of all announced projects, so that the agent can only conceal projects but not lie about projects she has available. To illustrate, consider the (implausible) situation in which the principal knows for sure that the agent has two projects to choose from. Then, if able to inspect both projects for free, the principal could require the agent to bring them both forward, and simply implement his preferred one and so obtain his first-best outcome. However, such "forcing" mechanisms cannot be used when the number of projects is uncertain (as is much more plausible), and they also cannot be used if the agent can fabricate additional related projects. ${ }^{19}$

\section{Searching for a Project}

The previous model assumed that the number of projects was exogenous to the agent (but uncertain). We now suppose instead that the agent can influence the number of available projects in a dynamic search framework. In particular, the rate of arrival of projects depends on privately costly search effort by the agent. Special cases of our formulation are standard search models in which: (i) the agent can instantaneously obtain a new draw by incurring a $\operatorname{cost} C$, and (ii) the agent is passive and must wait for a new draw to materialize exogenously.

As before, the agent's payoff (excluding search costs) is $u$, the principal's payoff is a weighted sum of the agent's payoff (including search costs) and the expected value of a random variable $v$, where the relative weight on the agent's payoff by the principal is $\alpha \leq 1$. The principal determines a function $r(\cdot)$ such that any type- $u$

\footnotetext{
${ }^{18}$ If the chosen project turns out not to have the claimed characteristics, suppose the principal does not implement any project.

${ }^{19}$ In the merger context it is easy to imagine that a firm with a particular merger possibility could also construct a second artificial merger option which is slightly less good for both consumers and profit. Such gaming is never worthwhile when the agent is simply faced with a permission set.
} 
project with $v \geq r(u)$ is permitted. ${ }^{20}$ Also as before, $x(u)$ is the probability that a project drawn at random either has agent utility below $u$ or is not in the permitted set.

Provided that search is worthwhile, the agent will keep searching until she finds a permitted project which delivers her utility $u$ above some threshold, denoted $U$, to be characterized below. The agent also decides how much effort to put into searching. Specifically, suppose that the arrival of projects follows a Poisson process, and a new project emerges with probability $h \times d t$ in any small time interval $d t$, where the hazard rate $h$ has associated private flow cost for the agent given by $c(h)$. Here, $c(\cdot)$ is assumed to be increasing and convex. To avoid corner solutions, suppose that $c^{\prime}(0)=0$. (However, we do not require that $c(0)=0$.) For use later, define $C$ to be the minimum average search cost, so that $C$ is the minimum value of $c(h) / h$ over $h \geq 0$.

The probability that a given project will be implemented is $1-x(U)$, while the agent's expected payoff at the time the project is implemented is $B(U) /(1-x(U))$, where

$$
B(U)=\int_{U}^{u_{\max }} u[1-G(r(u), u)] f(u) d u .
$$

Following this strategy, the agent will receive an acceptable project in a time interval $d t$ with probability $h(1-x(U)) \times d t$. This implies that the probability the first acceptable project will arrive in the time interval $(t, t+d t)$ is

$$
h(1-x(U)) e^{-h(1-x(U)) t} \times d t .
$$

If the agent discounts at the rate $\delta$, her expected utility is therefore

$$
\int_{0}^{\infty}\left[h(1-x(U)) \frac{B(U)}{(1-x(U))}-c(h)\right] e^{-h(1-x(U)) t} e^{-\delta t} d t=\frac{h B(U)-c(h)}{h(1-x(U))+\delta} .
$$

The agent will choose her reservation utility $U$ and search intensity $h$ in order to maximize her payoff (23). The first-order condition for choosing $U$ is

$$
U=\frac{h B(U)-c(h)}{h(1-x(U))+\delta} .
$$

In particular, $U$, the reservation utility, is also the agent's discounted payoff from following her optimal strategy (as is usual in search models). Using expression (24), the first-order condition for $h$ is

$$
h c^{\prime}(h)-c(h)=\delta U .
$$

Given that $c(\cdot)$ is convex, it follows from (25) that higher $U$ is associated with greater search effort (at least when $\delta>0$ ). Expression (25) represents the fundamental

\footnotetext{
${ }^{20}$ The discussion in section 3.3 of more ornate mechanisms in the choosing model also applies to this search model. In particular, in this search model it would be interesting in future work to investigate whether the principal can sometimes do better by making the threshold rule depend on the delay before a project is chosen. For instance, could it be optimal to impose a deadline on the agent?
} 
relationship between the agent's utility and her search effort. Since a high search effort benefits the principal as well as the agent, the principal has a reason (beyond the weight $\alpha$ placed on the agent's interests) to increase the agent's payoff.

Note that (24) and (25) imply that

$$
\int_{U}^{u_{\max }}(u-U)(1-G(r(u), u)) f(u) d u=c^{\prime}(h) .
$$

Expression (25) shows $h$ to be an increasing function of $U$, whereas (26) gives $h$ to be a decreasing function of $U$. Therefore, the pair of equations (25)-(26) can have at most one solution in $(U, h)$. It is clear that $h$ corresponding to $U=u_{\max }$ is smaller in (26) than it is in (25). The value of $h$ corresponding to $U=0$ in (25) is the $h$ which minimizes $c(h) / h$, say $h^{*}$. The $h$ corresponding to $U=0$ in $(26)$ is higher than $h^{*}$ provided that

$$
\int_{0}^{u_{\max }} u(1-G(r(u), u)) f(u) d u \geq c^{\prime}\left(h^{*}\right)=C,
$$

where the equality follows from the fact that $h^{*}$ implements the minimum average cost, $C$. Condition (27) simply states that the agent has an incentive to engage in search at all when faced with the permission rule $r(\cdot)$. Thus, whenever $(27)$ holds, there exists a unique solution to the pair of equations (25)-(26).

Finally, the comparative statics of the agent's strategy are clear-cut. If $\delta$ is increased, this shifts upwards the increasing relationship between $h$ and $U$ in (25) but leaves the relationship in (26) unaltered, and this therefore induces $U$ to fall and $h$ to rise (weakly). Likewise, if the principal shifts the permission rule $r(\cdot)$ upwards, this shifts downwards the relationship between $h$ and $U$ given in (26), but leaves (25) unaltered, and so induces the agent to lower both $U$ and $h$. This discussion is summarized in the following result:

Lemma 4 Suppose that the permission rule $r(\cdot)$ satisfies inequality (27). Then the agent is willing to search for a project, and her reservation utility $U$ and search effort effort $h$ are the unique solution to the pair of equations (25)-(26) (or, equivalently, to the pair of equations (24)-(25)). All else equal, (i) increasing $\delta$ induces the agent to (weakly) reduce $U$ and increase $h$, and (ii) shifting the rule $r(\cdot)$ upwards induces the agent to (weakly) reduce $U$ and $h$.

Turning to the principal's problem, let $Z$ denote the discounted expected value of $v$ :

$$
Z=\frac{h \int_{U}^{u_{\max }}\left(\int_{r(u)}^{v_{\max }} v g(v, u) d v\right) f(u) d u}{h(1-x(U))+\delta} .
$$

The principal aims to choose the permission rule $r(\cdot)$ and the agent's search strategy $(U, h)$ in order to maximize $\alpha U+Z$ subject to the agent's twin incentive constraints (24) and (25). The relationship between $h$ and $U$ in (25) cannot be influenced by the principal, although the principal can choose the point on this locus by choosing $r(\cdot)$ appropriately. 


\subsection{The optimality of linear rules}

We attempt to solve the principal's problem in two stages. First, for given reservation utility $U$, and hence a given $h$ satisfying (25), we derive the permission rule $r(u)$ which maximizes the principal's payoff subject to the single constraint (24). Subsequently, the optimal choice for $U$ can be analyzed. In this section, we consider the first sub-problem, which turns out to have a surprisingly simple solution.

Therefore, fix $U$ (and hence $h$ ). Writing $\gamma$ for the Lagrange multiplier on the constraint (24), the principal chooses $r(\cdot)$ to maximize the Lagrangian

$$
\mathcal{L}=Z+\gamma \frac{h B-c(h)}{h(1-x(U))+\delta}=\frac{h \int_{U}^{u_{\max }}\left(\int_{r(u)}^{v_{\max }}[v+\gamma u] g(v, u) d v\right) f(u) d u-c(h)}{h(1-x(U))+\delta}
$$

(The multiplier $\gamma$ will then be chosen in order to ensure that (24) binds.) For a given $U$ there are many ways to choose the rule $r(\cdot)$ which lead to a specified value of $1-x(U)$, the chance that a project is permitted and yields agent utility above the reservation level $U$. For a specified value of $1-x(U)$, it is clear that the way to maximize the numerator of the Lagrangian (29) is to set $r(u)=t-\gamma u$ for some $t$ which ensures that the target $1-x(U)$ is achieved. This simple result tells us immediately that the optimal rule $r(\cdot)$ will be linear in this search setting, regardless of the distribution of $(u, v)$.

Altering $t$ will affect both the numerator and denominator in the Lagrangian. Note that a small increase in $t$ loses $(v, u)$ points such that $v+\gamma u \approx t$. It follows that

$$
\frac{d}{d t} \int_{U}^{u_{\max }}\left(\int_{t-\gamma u}^{v_{\max }}[v+\gamma u] g(v, u) d v\right) f(u) d u=t \frac{d}{d t}(1-x(U)) .
$$

Differentiating (29) with respect to $t$ therefore implies that the optimal $t$ satisfies

$$
t=Z+\gamma U \text {. }
$$

Therefore $\mathcal{L}$ in (29) is maximized, given $U$ and $h$, when

$$
r(u)=Z-\gamma(u-U) \text {. }
$$

We summarize this discussion in the following result:

Proposition 5 The optimal strategy for the principal in the search framework is to offer a linear threshold rule (30), where $U$ is the agent's reservation utility and $Z$ is the expected discounted value of $v$.

The intuition for the, perhaps surprising, linearity of $r(u)$ comes from noting that for given $U$ and for given $1-x(U)$, the principal's problem is simply to maximize the expected value of the weighted sum $v+\gamma u$, where the weight $\gamma$ is the Lagrange multiplier on the constraint that the agent has reservation utility $U$.

General analysis of the second stage of the principal's problem, the analytic derivation of the optimal value of $U$, turns out to be complex. Instead, in the next two sections we focus on two polar cases: the case of no discounting (which is equivalent to the standard search problem where the agent can instantaneously obtain a new draw of project in return for a cost $C$ ), and the case of "urgent" projects where $\delta$ is large. 


\subsection{No discounting}

In this section we focus on the special case where $\delta=0$. The agent's choice of search effort cannot then be influenced by the principal (so long as the agent is willing to search at all). From expression (23), the agent will choose $U$ and $h$ in order to maximize

$$
\frac{B(U)-c(h) / h}{1-x(U)}
$$

and so $h$ is chosen to minimize $c(h) / h$ regardless of $r(\cdot)$. Since $C$ is the minimal value of $c(h) / h$, the agent will choose $U$ in order to maximize $[B(U)-C] /[1-x(U)]$. To make the problem interesting, suppose that $c(0)>0$ so that the agent faces a flow cost even to search at a minimal level. ${ }^{21}$ This implies that $C>0$. Note that this problem is formally identical to the problem where the agent can instantaneously obtain a new draw of project in return for a cost $C .^{22}$

When $\delta=0$, then $\gamma>0$ in (30) and the linear permission rule is always downward sloping. To see this, note from (28) that the equilibrium $Z$ satisfies

$$
0=\int_{U}^{u_{\max }}\left(\int_{Z-\gamma(u-U)}^{v_{\max }}[v-Z] g(v, u) d v\right) f(u) d u .
$$

If $\gamma \leq 0$ then the integrand above is always positive, and so the integral cannot equal zero. Thus, we must have $\gamma>0$ as claimed. The multiplier $\gamma>0$ is chosen to make constraint (24), or equivalently constraint (26), bind, so that

$$
\left.C=\int_{U}^{u_{\max }}(u-U)\right)(1-G(Z-\gamma(u-U), u)) f(u) d u .
$$

(Note that since $C$ is the minimum value of $c(h) / h$, it follows that $C=c^{\prime}(h)$.)

To obtain some explicit solutions to the principal's problem, suppose again that $(u, v)$ is uniformly distributed on the rectangle $[0,1] \times[-1,1]$. Expressions $(31)$ and (32) become respectively

$$
0=\frac{1}{12}(1-U)\left(3(1-Z)^{2}-\gamma^{2}(1-U)^{2}\right), \text { or } 1-Z=\frac{\gamma}{\sqrt{3}}(1-U),
$$

and

$$
C=\frac{1}{12}(1-U)^{2}(3(1-Z)+2 \gamma(1-U)) .
$$

Eliminating $\gamma$ from this pair of equations shows that

$$
Z=1-\frac{2 C}{\kappa(1-U)^{2}}
$$

\footnotetext{
${ }^{21}$ If $c(0)=0$, then $C=0$ and the problem is trivial. If $C=0$, the agent will make (almost) no search effort, and the principal will wait for an (almost) perfect project to materialize. Thus, there is almost no discretion and $r(u) \approx v_{\max }$. An example of this phenomenon can be seen in Figures 6 , 7 and 8 below.

${ }^{22}$ If the agent has search effort $h$, the expected time until a new project arrives is $1 / h$. Without discounting, therefore, the (expected) total cost of obtaining a new draw of project is $c(h) / h$. Once the agent minimizes this total cost, the cost of getting a new draw is $C$.
} 
where

$$
\kappa \equiv \frac{1}{2}+\frac{1}{\sqrt{3}} \approx 1.08
$$

Since the principal's payoff is $Z+\alpha U$, the principal will therefore choose $U$ to maximize

$$
\alpha U-\frac{2 C}{\kappa(1-U)^{2}} .
$$

This is a decreasing function of $U$ whenever

$$
\alpha \leq \frac{4 C}{\kappa} \approx 3.7 C
$$

in which case it is optimal to set $U=0$ and so leave the agent with zero rent. The optimal permission set is then $r(u)=Z-\gamma u$, where $Z$ is given by (34) and $\gamma$ is given by (33), both with $U$ set equal to zero. It follows that ${ }^{23}$

$$
r(u)=1-\frac{2 C}{\kappa}(u \sqrt{3}+1)
$$

for all $\alpha$ that satisfy (35). Thus, for different values of $C$ expression (36) traces out a family of linear, downward-sloping lines for $r(u)$. See Figure 6 for the case $\alpha=0$ (when condition (35) is always satisfied), where smaller $C$ correspond to higher $r$. When $C \approx 0$, we have $r(u) \approx v_{\max }$ as expected. (This is like the "large $\mu$ " case in section 3.2.)

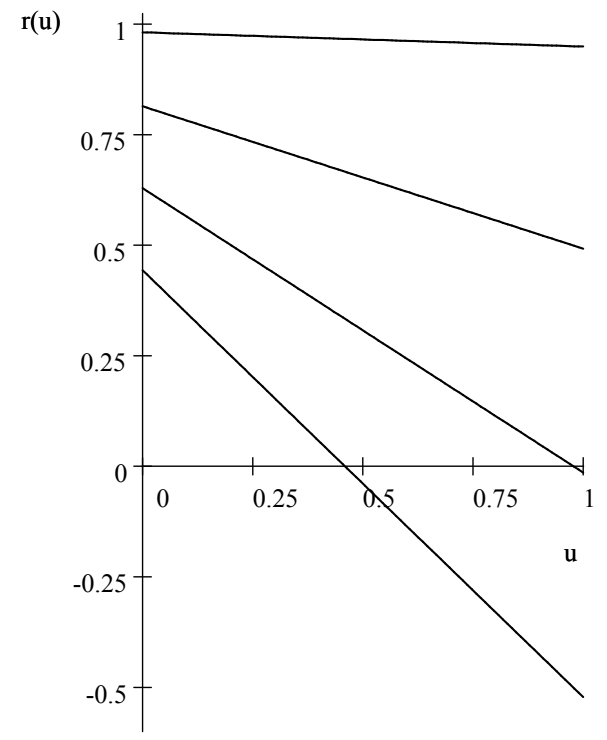

Figure 6: $r(u)$ for Uniform example with $\alpha=0$ and $C=0.01,0.1,0.2$ and 0.3

\footnotetext{
${ }^{23}$ These solutions are valid only when $C \leq \kappa /(1+\sqrt{3}) \approx 0.4$. This is to ensure that $r(u)$ does not hit the lower boundary $v=-1$. If $C>0.4$, the optimum will involve $r(u)$ being a downward-sloping linear function which hits this lower boundary (and $r(u) \equiv-1$ beyond this point).
} 
In the two models we have considered, with $\alpha=0$ and a uniform distribution for $(u, v)$, we have derived two surprisingly simple families of threshold rules (see (19) and (36) above). In one respect optimal policy is similar in the two models: as projects are easier to come by for the agent (i.e., $\mu$ is larger in the first model or $C$ is smaller in this second model), the permitted set of projects becomes progressively more restricted. In other respects, though, policy is dramatically different in the two settings. In the "choosing a project" model in section 3 , the rules start at $r(0)=0$ and increase, and only desirable projects (i.e., $v \geq 0$ ) are permitted. In the "searching" model we have $r(0)>0, r$ is decreasing, and it may be optimal to permit projects with negative payoff for the principal (as when $C=0.3$ in Figure 6).

The downward slope of $r(u)$ in the "searching" model when $\alpha=0$ can be explained as follows. The principal wishes to maximize the expected value of $v$ in the permitted set, subject to the agent being willing to engage in costly search for permitted projects. For a given expected value of $v$ in the permitted set, the principal is indifferent about whether the threshold rule is upward or downward sloping; however, the agent's willingness to search is enhanced when higher- $u$ projects are more likely to be permitted, i.e., when the rule is downward sloping. For the same reason, it can be optimal to permit the agent to choose projects with a negative payoff for the principal, if the search cost is large enough.

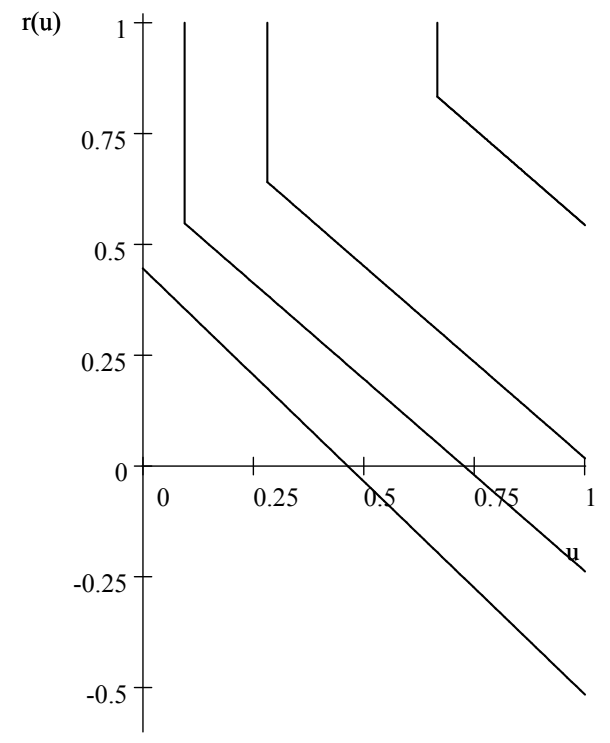

Figure 7: $r(u)$ for Uniform example with $\alpha=1$ and $c=0.01,0.1,0.2$, and 0.3

If (35) does not hold then $U>0$ is optimal. Indeed optimal $U$ and $\gamma$ satisfy

$$
1-U=\left(\frac{4 C}{\kappa \alpha}\right)^{\frac{1}{3}}, \gamma=\frac{\sqrt{3}}{2} \alpha .
$$

Note that $\gamma$ here is independent of $C$ and less than $\alpha$. Therefore, the permission rule is

$$
r(u)=1-(1+\sqrt{3})\left(\frac{C \alpha^{2}}{2 \kappa}\right)^{\frac{1}{3}}+\frac{\sqrt{3}}{2} \alpha(1-u) .
$$


In Figure 7 we show the permission sets when $\alpha=1$, for the same search costs as in Figure 6. Since the agent will keep searching until a permitted project with $u>U$ is found, only that part of the rule with $u>U$ is relevant, and that part is depicted on the figure. (The principal can choose the linear rule without constraining $u>U$, so that the downward-sloping lines can be extended to the left until they reach the vertical axis, but the agent will never choose a permitted project to the left of the vertical lines shown.) When the principal wishes to maximize total welfare $(\alpha=1)$, Figure 7 suggests that a good approximation to optimal policy is to permit projects which increase total welfare by some discrete threshold, where this threshold is higher when projects are less costly to discover.

\subsection{The impact of discounting}

We now discuss cases with discounting, including the limit case where $\delta \rightarrow \infty$. For intermediate values of $\delta$, it seems necessary to use numerical methods with particular examples to proceed. To this end, consider again the example where $(u, v)$ is uniformly distributed on $[0,1 \times[-1,1]$. Then expressions $(26)$ and (28) become respectively

$$
\begin{aligned}
& c^{\prime}(h)=\frac{1}{12}(1-U)^{2}(3(1-Z)+2 \gamma(1-U)) \\
& \frac{\delta}{h} Z=\frac{1}{12}(1-U)\left(3(1-Z)^{2}-\gamma^{2}(1-U)^{2}\right) .
\end{aligned}
$$

Suppose that the effort function is $c(h)=h^{2}$, in which case expression (25) implies

$$
h=\sqrt{\delta U} .
$$

Eliminating $\gamma$ from the two equations (38)-(37) and substituting for the value of $h$ in (39) yields the following (tedious) expression for $Z$ in terms of the agent utility $U$ :

$$
Z=1-4 \frac{\sqrt{(1-U)^{3} \sqrt{U / \delta}+4\left(4 U+7 U^{2}+1\right)}-4 U-2}{(1-U)^{2} \sqrt{U / \delta}}
$$

To find the optimal permission rule corresponding to a particular value of $\delta$ in this example, one can work as follows. The principal wishes to choose $U$ to maximize $Z+$ $\alpha U$, where $Z$ is given by expression (40). For given $\delta$ this can be done (numerically), and then the value of the slope $\gamma$ of $r(u)$ can be obtained from expression (37). The permission rules for various values of $\delta$ are illustrated in this figure for the case $\alpha=0$. (Larger $\delta$ correspond to lower permission rules in the figure.)

As is intuitive, smaller values of delta lead to a higher payoff for the principal. (The payoffs $(U, Z)$ to the two parties are given by the "corners" of the permission rules in the figure.) In the limit as $\delta \rightarrow 0$, the principal can obtain almost the maximum possible payoff of 1 by permitting the agent to choose only those projects which are almost the best possible for the principal; the agent will expend minimal 
(but positive) effort and eventually a qualified project will emerge. ${ }^{24}$ Note that the optimal permission rules have upward slope when $\delta$ is very small.

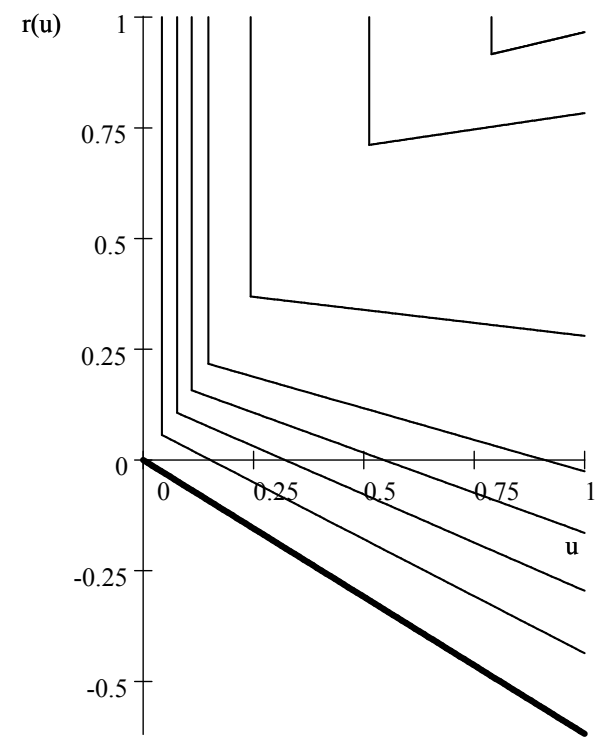

Figure 8: $r(u)$ for $\delta=10^{-7}, 10^{-4}, 0.01,0.05,0.1,0.2,0.5$, and $\delta=\infty$ (in bold)

In this example, when $\delta$ is larger the principal finds it optimal to induce more search effort from the agent. ${ }^{25}$ This implies that the principal expects to wait a shorter time for a permitted project (acceptable to the agent) to appear. ${ }^{26}$ However, since the permission rule $r(\cdot)$ is lower when $\delta$ is higher, the principal's expected payoff when an acceptable project emerges is lower when $\delta$ is higher. Thus, as is intuitive, the principal is willing to accept a less good project, but with less delay, when discounting is more significant.

The limit of impatience as $\delta$ becomes large is straightforward to analyze in general terms. From (23) and (28), $U$ and $Z$ tend to zero as $\delta \rightarrow \infty$. Therefore, Proposition 5 indicates that the rule $r(\cdot)$ will be a straight line emanating from $(0,0)$. However, it is perhaps more illuminating to derive the solution directly. The impact of the assumption that $\delta$ is large is that both principal and agent aim to maximize their flow payoff. Thus, faced with a particular rule $r(\cdot)$, the agent will choose $U$ and $\delta$ to maximize $h B(U)-c(h)$, where $B(U)$ is given in (22). It is immediate that the agent will choose $U=0$, and implement the first permitted project. Thus, if we write

\footnotetext{
${ }^{24}$ From the figure it is apparent that the agent also obtains almost the ideal payoff of 1 too. However, that is inessential to the solution. When $\delta \approx 0$, the curve (40) is almost flat and approximately equal to 1 for $U>0$, and so the principal could do almost as well by giving the agent minimum rent as by giving her maximal rent. Of course, if $\alpha>0$ then the principal has a strict incentive to offer high rent to the agent.

${ }^{25}$ From (39), with $\delta=10^{-7}$ effort $h$ is about 0.0003 , and this increases monotonically to $h=0.15$ when $\delta=0.5$.

${ }^{26}$ The expected waiting time for an acceptable project to appear is $(h(1-x(U)))^{-1}$, and this decreases with $\delta$ since both $h$ and $1-x(U)$ increase with $\delta$ in this example.
} 
$B=B(0)$ the agent will choose $h$ to maximize $h B-c(h)$. If we write

$$
\sigma(B) \equiv \max _{h}: h B-c(h)
$$

then $\sigma$ is a convex increasing function and $\sigma^{\prime}(B)$ is the agent's choice of effort given her reward $B$.

The principal chooses $r(\cdot)$ to maximize his flow payoff:

$$
h \int_{0}^{u_{\max }}\left(\int_{r(u)}^{v_{\max }} v g(v, u) d v\right) f(u) d u+\alpha(h B-c(h)),
$$

which can be written as

$$
\sigma^{\prime}(B) A+\alpha \sigma(B)
$$

where

$$
A=\int_{0}^{u_{\max }}\left(\int_{r(u)}^{v_{\max }} v g(v, u) d v\right) f(u) d u .
$$

By considering small changes in $r(\cdot)$ at $u$, it follows that (41) is maximized when

$$
r(u)+\left[A \frac{\sigma^{\prime \prime}(B)}{\sigma^{\prime}(B)}+\alpha\right] u \equiv 0,
$$

and $r$ is indeed a straight line starting at the origin. ${ }^{27}$ Moreover, the line is downward sloping and weakly steeper than the principal's true preferences (which have slope $-\alpha$ ). The only situation in which the principal implements his naive rule, i.e., $r(u)=-\alpha u$, is when $\sigma^{\prime \prime}=0$, which applies when the agent's search effort does not respond to incentives, i.e., there is an exogenous hazard rate. Outside this dull case, though, in this limit of impatience the principal allows some projects which are strictly undesirable $(v+\alpha u<0)$ in order to stimulate search effort by the agent. ${ }^{28}$ This distortion is the opposite to the bias in the "choosing" model, where the principal forbade some desirable projects.

\footnotetext{
${ }^{27}$ For example, take the case where $c(h)=a+\frac{1}{2} b h^{2}$. Then $\sigma(B)=\frac{1}{2 b} B^{2}$ and (42) becomes

$$
r(u)=-\left(\frac{A}{B}+\alpha\right) u .
$$
}

This permission rule does not depend on the specific parameters of the quadratic cost function. In the example depicted in Figure 8 above, (42) implies that $r(u)=-\frac{1}{2}(\sqrt{5}-1) u$, and this line is drawn in bold on that figure.

${ }^{28}$ This feature is also seen in Baker, Gibbons, and Murphy (1999). By contrast, as discussed in section 1, Szalay (2005) presents a model where "search incentives" are enhanced by forbidding projects which the principal and agent might often wish to implement. 


\section{Conclusions}

Proceeding from the motivating example of welfare standards in merger policy, we have explored the nature of optimal discretion for a principal to give to an agent in two related settings of delegated project choice with (contingent) transfers ruled out. The principal's problem is to design the optimal set of permitted projects without knowing which projects are available to the agent, though being able to verify the characteristics of the project chosen by the agent. In other words, the problem is to set the optimal rule that the agent must obey, in circumstances where the principal can just check whether or not the rule has been met.

In the first setting the agent has a number (unknown to the principal) of projects to choose from. The optimal permission set excludes some projects that are good for the principal because the loss from excluding marginally good projects is outweighed by the expected gain from thereby inducing the choice of better projects. We showed (i) the principal permits more types of project when he put more weight on the agent's welfare, and (ii) the principal permits fewer types of project when the agent has more projects to choose from. Solutions for the optimal delegation set were derived for some examples.

In the second setting the agent searches for a project that is both permitted by the principal and meets the agent's own acceptance threshold. Here the optimal permission set was quite generally characterised by a linear relationship between the payoffs of principal and agent. In order to encourage search effort, or to cover fixed search costs efficiently, projects with higher agent payoffs are permitted for a wider range of principal payoffs, so that the permission rule is downward sloping. In order to encourage search, the principal might permit some projects which are undesirable ex post (especially with high discounting), in contrast to the bias induced in the "choosing" model.

Our two settings have therefore illustrated different aspects of the problem of optimally constraining the choices of an agent when the principal can simply commit to what the agent is and is not allowed to do. Elements of our analysis could perhaps be extended to situations where the principal has richer information (e.g., about unchosen projects) and/or more instruments (e.g., some scope to make contingent transfers or to impose deadlines on the agent). Another way to develop the analysis could be to multi-agent settings: it is after all a feature of many rules that they apply without discrimination to various agents in various situations. 


\section{APPENDIX: Derivation of the Euler equation (6)}

The following analysis follows the argument in any standard textbook on the calculus of variations. Write

$$
s(p, u) \equiv \int_{r(p, u)}^{v_{\max }} v g(v, u) d v
$$

where $r(p, u)$ is defined implicitly by

$$
G(r(p, u), u) \equiv 1-p
$$

Thus $r(p, u)$ is the threshold such that a proportion $p$ of projects lie above $r(p, u)$ for given $u$, and $s(p, u)$ is the sum of $v$ above this threshold. Therefore, $V(r(p, u), u)=$ $s(p, u) / p$. Note that $s$ is increasing and concave in $p$, and ranges from 0 to $E[v \mid u]$ as $p$ ranges from 0 to 1 . Note also that $s_{p}(p, u) \equiv r(p, u)$. Viewing $p$ as a function of $u$, we have $x^{\prime}(u)=f(u) p(u)$ as in (1) above.

The principal's aim is to maximize

$$
\int_{0}^{u_{\max }}[V+\alpha u] \frac{d}{d u} \phi(x(u)) d u=\int_{0}^{u_{\max }}\left[f(u) s\left(\frac{x^{\prime}(u)}{f(u)}, u\right)+\alpha u x^{\prime}(u)\right] \phi^{\prime}(x(u)) d u
$$

subject to the endpoint condition $x\left(u_{\max }\right)=1$. Write

$$
H\left(u, x, x^{\prime}\right) \equiv\left[f(u) s\left(\frac{x^{\prime}}{f(u)}, u\right)+\alpha u x^{\prime}\right] \phi^{\prime}(x)
$$

for the Hamiltonian for this problem.

Let $\eta(u)$ by any smooth function such that $\eta\left(u_{\max }\right)=0$, and consider the function

$$
\Psi(t)=\int_{0}^{u_{\max }} H\left(u, x+t \eta, x^{\prime}+t \eta^{\prime}\right) d u
$$

If $x$ is the optimal path, it follows that $\Psi(t)$ must be maximized at $t=0$, for any $\eta$. By Leibniz's rule

$$
\Psi^{\prime}(0)=\int_{0}^{u_{\max }}\left[\eta H_{x}\left(u, x, x^{\prime}\right)+\eta^{\prime} H_{x^{\prime}}\left(u, x, x^{\prime}\right)\right] d u .
$$

Integrating the second term by parts yields

$$
\left.\int_{0}^{u_{\max }} \eta^{\prime} H_{x^{\prime}} d u=\eta H_{x^{\prime}}\right]_{0}^{u_{\max }}-\int_{0}^{u_{\max }} \eta \frac{d}{d u} H_{x^{\prime}} d u .
$$

The only way that the problem can be stationary at $x$ is if $H_{x^{\prime}}=0$ when $u=0$ (for otherwise we could choose $\eta(0)$ to be any value which makes the above very large). Since $H_{x^{\prime}}=\phi^{\prime}(x) f(0) s_{p}\left(x^{\prime} / f(0), 0\right)$ when $u=0$, it follows that at the optimum we must have $s_{p}(p(0), u)=r(0)=0$, and assume this henceforth. 
It follows that

$$
\Psi^{\prime}(0)=\int_{0}^{u_{\max }} \eta\left\{H_{x}-\frac{d}{d u} H_{x^{\prime}}\right\} d u .
$$

Since this must be zero for all deviations $\eta(\cdot)$, it follows that $\{\cdot\}$ must be zero for all $u$, and we obtain the central Euler equation

$$
H_{x}\left(u, x, x^{\prime}\right)=\frac{d}{d u} H_{x^{\prime}}\left(u, x, x^{\prime}\right) .
$$

Written explicitly this becomes

$$
\phi^{\prime \prime}(x)\left[f s\left(\frac{x^{\prime}}{f}, u\right)+\alpha u x^{\prime}\right]=\phi^{\prime}(x) \frac{d}{d u}\left[s_{p}\left(\frac{x^{\prime}}{f}, u\right)+\alpha u\right]+\phi^{\prime \prime}(x) x^{\prime}\left[s_{p}\left(\frac{x^{\prime}}{f}, u\right)+\alpha u\right] \text {. }
$$

Since $p(u)=x^{\prime}(u) / f(u)$ and $r(u) \equiv s_{p}(p(u), u)$, this expression becomes

$$
r^{\prime}(u)+\alpha=\frac{\phi^{\prime \prime}}{\phi^{\prime}} f p\left[\frac{s(p, u)}{p}-r\right]
$$

which leads to expression (6) in the text.

What about the second-order condition? When $H$ is jointly concave in $x$ and $x^{\prime}$, it is known that the solution to the Euler equation is the global maximum. However, since $H_{x x}=\left[f s\left(x^{\prime} / f, u\right)+\alpha u x^{\prime}\right] \phi^{\prime \prime \prime}(x)>0$ this sufficient condition never applies in our problem. Therefore, we look for local second-order conditions. Repeated differentiation yields

$$
\Psi^{\prime \prime}(0)=\int_{0}^{u_{\max }}\left[\eta^{2} H_{x x}+2 \eta \eta^{\prime} H_{x x^{\prime}}+\left(\eta^{\prime}\right)^{2} H_{x^{\prime} x^{\prime}}\right] d u
$$

It is well known that a necessary condition for $\Psi^{\prime \prime}(0)$ to be negative for all $\eta$ is that $H_{x^{\prime} x^{\prime}} \leq 0$ along the optimal path. (This is the "Legendre condition".) In our problem, $H_{x^{\prime} x^{\prime}}=\phi^{\prime}(x) s_{p p}\left(x^{\prime} / f, u\right) / f<0$ and so this necessary condition is indeed satisfied. But it would be reassuring to have a sufficient condition for $\Psi^{\prime \prime}(0)<0$ as well.

The middle term in the above integral can be integrated by parts to give

$$
\left.\int_{0}^{u_{\max }} 2 \eta \eta^{\prime} H_{x x^{\prime}} d u=\eta^{2} H_{x x^{\prime}}\right]_{0}^{u_{\max }}-\int_{0}^{u_{\max }} \eta^{2} \frac{d}{d u} H_{x x^{\prime}} d u=-\int_{0}^{u_{\max }} \eta^{2} \frac{d}{d u} H_{x x^{\prime}} d u .
$$

Here, the second equality follows from the observation that $H_{x x^{\prime}}=0$ when $u=0$ (and also that $\left.\eta\left(u_{\max }\right)=0\right)$. Therefore,

$$
\Psi^{\prime \prime}(0)=\int_{0}^{u_{\max }} \eta^{2}\left[H_{x x}-\frac{d}{d u} H_{x x^{\prime}}\right]+\left(\eta^{\prime}\right)^{2} H_{x^{\prime} x^{\prime}} d u .
$$

Since $H_{x^{\prime} x^{\prime}}<0$, a sufficient condition for $\Psi^{\prime \prime}(0)<0$ for all $\eta$ is that the above term [.] be weakly negative along the optimal path. Writing explicitly shows that

$$
H_{x x}-\frac{d}{d u} H_{x x^{\prime}}=\phi^{\prime \prime \prime} f p\left(\frac{s}{p}-s_{p}\right)-\phi^{\prime \prime}\left(r^{\prime}+\alpha\right)=f p\left(\frac{s}{p}-s_{p}\right)\left(\phi^{\prime \prime \prime}-\phi^{\prime \prime} \frac{\phi^{\prime \prime}}{\phi^{\prime}}\right),
$$

where the second equality follows from (44). Since $s$ is concave in $p$ it follows that $\left(\frac{s}{p}-s_{p}\right)$ is positive, and so the above expression is weakly negative if and only if $\zeta(x) \equiv \phi^{\prime \prime}(x) / \phi^{\prime}(x)$ weakly decreases with $x$. In such cases, we may be sure that the second-order condition $\Psi^{\prime \prime}(0)<0$ is satisfied. 


\section{References}

Aghion, P., And J. Tirole (1997): "Formal and Real Authority in Organizations," Journal of Political Economy, 105(1), 1-29.

Alonso, R., and N. Matouschek (2007): "Relational Delegation," RAND Journal of Economics, 38(4), 1070-1089.

(2008): "Optimal Delegation," Review of Economic Studies, 75(1), 259-294.

Amador, M., I. Werning, and G.-M. Angeletos (2006): "Commitment vs. Flexibility," Econometrica, 74(2), 365-396.

Baker, G., R. Gibbons, and K. Murphy (1999): "Informal Authority in Organizations," Journal of Law, Economics and Organization, 15(1), 56-73.

Falk, A., And M. Kosfeld (2006): "The Hidden Costs of Control," American Economic Review, 96(5), 1611-1630.

FArrell, J., And M. Katz (2006): "The Economics of Welfare Standards in Antitrust," Competition Policy International, 2(2), 3-28.

Fridolfsson, S.-O. (2007): "A Consumer Surplus Defense in Merger Control," in The Political Economy of Antitrust, ed. by V. Ghosal, and J. Stennek, pp. 287-302. Elsevier, Amsterdam.

Holmstrom, B. (1984): "On the Theory of Delegation," in Bayesian Models in Economic Theory, ed. by M. Boyer, and R. Kihlstrom. Elsevier, Amsterdam.

Holmstrom, B., And P. Milgrom (1991): "Multi-Task Principal-Agent Analysis: Incentive Contracts, Asset Ownership and Job Design," Journal of Law, Economics and Organization, 7(1), 24-52.

Kovác, E., and T. Mylovanov (2007): "Stochastic Mechanisms in Settings without Monetary Transfers: The Regular Case," mimeo, University of Bonn.

Lyons, B. (2002): "Could Politicians Be More Right Than Economists? A Theory of Merger Standards," mimeo, University of East Anglia.

Martimort, D., And A. Semenov (2006): "Continuity in Mechanism Design without Transfers," Economics Letters, 93(2), 182-189.

Melumad, N., and T. Shibano (1991): "Communication in Settings with no Transfers," RAND Journal of Economics, 22(2), 173-198.

SzAlay, D. (2005): "The Economics of Clear Advice and Extreme Options," Review of Economic Studies, 72(4), 1173-1198. 